\title{
The volume product of convex bodies with many hyperplane symmetries
}

\author{
F. Barthe and M. Fradelizi
}

\section{To appear in Amer. J. Math.}

\begin{abstract}
Mahler's conjecture predicts a sharp lower bound on the volume of the polar of a convex body in terms of its volume. We confirm the conjecture for convex bodies with many hyperplane symmetries in the following sense: their hyperplanes of symmetries have a one-point intersection. Moreover, we obtain improved sharp lower bounds for classes of convex bodies which are invariant by certain reflection groups, namely direct products of the isometry groups of regular polytopes.
\end{abstract}

Mathematic Subject Classification: 52A20; 52A40; 52A38; 52B15; 46B10

\section{Introduction}

We work in $\mathbb{R}^{n}$ equipped with its canonical Euclidean structure. The scalar product is denoted by $\langle\cdot, \cdot\rangle$ and the norm by $|\cdot|$. Let $K$ be a convex body in $\mathbb{R}^{n}$. For $z \in \mathbb{R}^{n}$, the polar body of $K$ with respect to $z$ is defined by

$$
K^{z}=\left\{y \in \mathbb{R}^{n} ;\langle y-z, x-z\rangle \leq 1 \text { for every } x \in K\right\} .
$$

We write $K^{\circ}$ for $z=0$, which should not be confused with $\operatorname{Int}(K)$, the interior of $K$. In what follows, the volume of a convex set $K \subset \mathbb{R}^{n}$ is denoted by $|K|_{n}$ and simply $|K|$ when there is no ambiguity. The volume product of $K$ is by definition

$$
\mathcal{P}(K):=\inf _{z \in \mathbb{R}^{n}}|K|\left|K^{z}\right| .
$$

It is known (see [15]) that the above infimum is achieved for a unique point, called the Santaló point of $K$ and denoted by $s(K)$. The volume product and the Santaló point are affine-invariant in the sense that for every non singular affine transform $A$, one has $\mathcal{P}(A K)=\mathcal{P}(K)$ and $s(A K)=A s(K)$. Hence if there are affine maps which fix $K$ globally and whose only common fixed point is the origin then $s(K)=0$ and $\mathcal{P}(K)=|K|\left|K^{\circ}\right|$. This is the case in particular if $K$ is origin-symmetric (i.e. $K=-K$ ). A celebrated result of Blaschke ([1] for $n=2$ or 3) and Santaló ([24] for general $n$ ) asserts that among $n$-dimensional convex bodies, the Euclidean ball has maximal volume product. The so-called Mahler's conjecture (see [12]) asserts that, among convex bodies having a center of symmetry, the volume product should be minimal for the cube (and also for its dual and for some combinations of the two sets, see the end of section 5 for more details), equivalently, the conjecture predicts that for such a convex body $K \subset \mathbb{R}^{n}$,

$$
|K|\left|K^{\circ}\right| \geq\left|B_{1}^{n}\right|\left|B_{\infty}^{n}\right|=\frac{4^{n}}{n !}
$$

where $B_{\infty}^{n}=[-1,1]^{n}$ and for $p \in(0, \infty), B_{p}^{n}=\left\{x \in \mathbb{R}^{n} ; \sum\left|x_{i}\right|^{p} \leq 1\right\}$. A corresponding question asks if among general convex bodies the volume product is minimal for simplices: is it true that for every convex body $K \subset \mathbb{R}^{n}$ and every $z \in \mathbb{R}^{n}$ the following inequality holds

$$
|K|\left|K^{z}\right| \geq\left|\Delta_{n}\right|\left|\left(\Delta_{n}\right)^{\circ}\right|=\frac{(n+1)^{n+1}}{(n !)^{2}},
$$


where $\Delta_{n}$ is a regular simplex in $\mathbb{R}^{n}$, with 0 as center of mass.

The conjectures were fully confirmed in dimension 2 by Mahler [13]. A few positive results were obtained in arbitrary dimension. The symmetric version of the conjecture was established for unconditional bodies by Saint Raymond [23] (see also Meyer [14] for a simple proof). Recall that a convex body in $\mathbb{R}^{n}$ is called unconditional if for all choices of signs $\varepsilon=\left(\varepsilon_{1}, \ldots, \varepsilon_{n}\right) \in\{-1,1\}$ we have

$$
x=\left(x_{1}, \ldots, x_{n}\right) \in K \Longrightarrow\left(\varepsilon_{1} x_{1}, \ldots, \varepsilon_{n} x_{n}\right) \in K .
$$

Equivalently, $S_{i}(K)=K$ for $i=1, \ldots, n$ where $S_{i}$ denotes the orthogonal symmetry with respect to the coordinate hyperplane $\left\{x \in \mathbb{R}^{n} ; x_{i}=0\right\}$. The conjecture was proved by Reisner [21] for zonoids (see also [8] for a simple proof) and by Meyer and Reisner [17] for polytopes with less than $n+3$ vertices. For convex bodies close, in Banach-Mazur distance, to the cube (resp. to the simplex) the inequalities were established by Nazarov, Petrov, Ryabogin and Zvavitch [19] (resp. by Kim and Reisner [10]).

An asymptotic version of the conjectures was also considered. Bourgain and Milman [2] established that there is a universal constant $c>0$ such that for every integer $n \geq 1$ and every convex body $K$ in $\mathbb{R}^{n}$,

$$
\mathcal{P}(K)^{\frac{1}{n}} \geq \frac{c}{n} .
$$

Other proofs were given by Pisier [20] and more recently by Kuperberg [11] and Nazarov [18].

Functional versions of Mahler's conjectures were also investigated by the second author and Meyer [5, 6, 7].

The aim of the present paper is to confirm Mahler's conjecture under rather general symmetry assumptions and to improve the conjectured bound in certain cases. The idea of the proof is to adapt Meyer's argument [14] for unconditional convex bodies to the case of more general symmetry assumptions.

To state our results more precisely, we need to introduce some notations. We denote the orthogonal group (the set of linear isometries of $\mathbb{R}^{n}$ ) by $\mathcal{O}\left(\mathbb{R}^{n}\right)$. For a subset $A \subset \mathbb{R}^{n}$, we define the group of linear isometries of $A$ as $\mathcal{O}(A)=\left\{g \in \mathcal{O}\left(\mathbb{R}^{n}\right) ; g(A)=A\right\}$. Also $\operatorname{span}(A), \operatorname{conv}(A)$ and $\operatorname{pos}(A)$ denote respectively the smallest vector space, the smallest convex set and the smallest convex cone containing $A$. Given a vector subspace $E \subset \mathbb{R}^{n}, P_{E}$ stands for the orthogonal projection onto $E$ and $S_{E}=2 P_{E}-\operatorname{Id}_{\mathbb{R}^{n}}$ for the orthogonal symmetry with respect to $E$. The orthogonal symmetry with respect to a hyperplane is also called a reflection. Eventually, if a group $G$ acts on $\mathbb{R}^{n}, \operatorname{Fix}(G)=\left\{x \in \mathbb{R}^{n} ; \forall g \in G, g x=x\right\}$ is the set of points which are fixed by the whole group.

The main results of this article are contained in the following theorem.

Theorem 1. Let $n \geq 2$ and $K \subset \mathbb{R}^{n}$ be a convex body.

(i) If $\mathcal{O}(K)$ contains $\mathcal{O}(P)$ for some regular polytope $P$ in $\mathbb{R}^{n}$ then $\mathcal{P}(K) \geq \mathcal{P}(P)$ with equality if and only if $K$ is a dilate of $P$ or $P^{\circ}$.

(ii) If $\mathcal{O}(K)$ contains $\mathcal{O}\left(P_{1}\right) \times \cdots \times \mathcal{O}\left(P_{k}\right)$ for some regular polytopes or Euclidean balls $P_{1}$ in $\mathbb{R}^{n_{1}}, \ldots, P_{k}$ in $\mathbb{R}^{n_{k}}$, with $n_{1}+\cdots+n_{k}=n$ then $\mathcal{P}(K) \geq \mathcal{P}\left(P_{1} \times \cdots \times P_{k}\right)$.

(iii) If $\mathcal{O}(K)$ contains $m$ reflections $S_{H_{1}}, \ldots, S_{H_{m}}$ with respect to $m$ hyperplanes $H_{1}, \ldots, H_{m}$ such that $\bigcap_{i=1}^{m} H_{i}=\{0\}$ then $\mathcal{P}(K) \geq \mathcal{P}\left(\Delta_{n}\right)$, with equality if and only if $K$ is a simplex.

Section 2 is devoted to convex sets in the plane. We establish the sharp lower bounds under symmetry assumptions and prove that regular polygons play an extremal role. This study allows to put forward some of the key ingredients which will be crucial in higher dimensions. In particular, the method requires an essential partition of the convex body $K$ of the form $K=\bigcup_{i} K \cap C_{i}$ where the sets $C_{i}$ are convex cones with the property that any point $y \in C_{i}$ is "normed" by a point of $K \cap C_{i}$ in the following sense

$$
\sup _{x \in K \cap C_{i}}\langle y, x\rangle=\sup _{x \in K}\langle y, x\rangle .
$$


Section 3 shows how to ensure this property when a convex body $K$ in arbitrary dimension has many symmetries. Section 4 deals with regular polytopes. The main result there is that a convex body having the same symmetries as a regular polytope has a larger volume product. This is a warm-up for Section 5 where a similar statement is established for convex bodies which are invariant by the direct product of the isometry groups of regular polytopes. Section 6 deduces from the result of Section 5 that Mahler's conjecture is indeed true for convex bodies having enough hyperplane symmetries. The classification of reflection groups plays a crucial role here. In section 7, we deduce Mahler's conjecture for some bodies of revolution, equivalently we establish functional forms of Mahler's conjecture for concave functions having many symmetries. The final section collects remarks and further questions.

\section{In the plane}

Given a convex body containing the origin it its interior, a natural strategy in order to evaluate $|K|\left|K^{\circ}\right|$ is to divide the plane into convex cones with apex at the origin and to work separately in each cone. This is possible only when $K$ meets the boundary of the cone in a certain way, as the next statement shows.

Proposition 2. Let $\alpha \in(0, \pi)$ and $u_{1}, u_{2}$ be unit vectors in $\mathbb{R}^{2}$ with angle $\alpha$, i.e. $\left\langle u_{1}, u_{2}\right\rangle=\cos \alpha$. Denote by $C:=\operatorname{pos}\left(u_{1}, u_{2}\right)$ the positive convex cone generated by $u_{1}, u_{2}$. Let $C_{i}:=\mathbb{R}^{+} u_{i}$ and $P_{i}$ the orthogonal projection onto $\mathbb{R} u_{i}$. Let $K$ be a convex body containing the origin in its interior, with the property that

$$
K \cap C_{i}=P_{i}(K) \cap C_{i}
$$

Then

$$
|K \cap C|_{2}\left|K^{\circ} \cap C\right|_{2} \geq \frac{1}{4}\left(2-\left(\frac{\left|K \cap C_{1}\right|_{1}}{\left|K \cap C_{2}\right|_{1}}+\frac{\left|K \cap C_{2}\right|_{1}}{\left|K \cap C_{1}\right|_{1}}\right) \cos \alpha\right),
$$

with equality if and only if $K \cap C=\operatorname{conv}\left(0, \lambda_{1} u_{1}, \lambda_{2} u_{2}\right)$ or $K \cap C=\left\{x \in C ;\left\langle x, u_{1}\right\rangle \leq \frac{1}{\lambda_{1}}\right.$ and $\left.\left\langle x, u_{2}\right\rangle \leq \frac{1}{\lambda_{2}}\right\}$ for some $\lambda_{1}, \lambda_{2}>0$ such that $\lambda_{1} \geq \lambda_{2} \cos \alpha$ and $\lambda_{2} \geq \lambda_{1} \cos \alpha$.

Proof. First let us comment on our hypothesis. Let $\lambda_{1}, \lambda_{2}>0$ such that $K \cap C_{i}=\left[0, \lambda_{i} u_{i}\right]$. The hypothesis $P_{i}(K) \cap C_{i}=K \cap C_{i}=\left[0, \lambda_{i} u_{i}\right]$ for $i \in\{1,2\}$ is equivalent to

$$
\left[0, \lambda_{i} u_{i}\right] \subset K \subset\left\{x ;\left\langle x, u_{i}\right\rangle \leq \lambda_{i}\right\} .
$$

Since, for $j \neq i \in\{1,2\}, \lambda_{j} u_{j} \in K$, we get that $\left\langle\lambda_{j} u_{j}, u_{i}\right\rangle \leq \lambda_{i}$ hence $\lambda_{j} \cos \alpha \leq \lambda_{i}$. Moreover taking polars, notice that the preceding inclusions are also equivalent to

$$
\left[0, \frac{u_{i}}{\lambda_{i}}\right] \subset K^{\circ} \subset\left\{x ;\left\langle x, \lambda_{i} u_{i}\right\rangle \leq 1\right\}
$$

and thus to $P_{i}\left(K^{\circ}\right) \cap C_{i}=K^{\circ} \cap C_{i}=\left[0, u_{i} / \lambda_{i}\right]$. Let $v_{1}, v_{2}$ be the inner (unit) normal vectors to the edges of $C$ so that $C=\left\{x ;\left\langle x, v_{i}\right\rangle \geq 0, i=1,2\right\}$. We follow the argument of Meyer [14]. For every $x \in K \cap C$

$$
|K \cap C| \geq \sum_{i=1}^{2}\left|\operatorname{conv}\left(x, K \cap C_{i}\right)\right|=\sum_{i=1}^{2} \frac{\left|K \cap C_{i}\right|\left\langle x, v_{i}\right\rangle}{2}=\frac{\left\langle x, \lambda_{1} v_{1}+\lambda_{2} v_{2}\right\rangle}{2} .
$$

This implies that the vector

$$
y_{0}:=\frac{\lambda_{1} v_{1}+\lambda_{2} v_{2}}{2|K \cap C|}=\frac{\left(\lambda_{2}-\lambda_{1} \cos \alpha\right) u_{1}+\left(\lambda_{1}-\lambda_{2} \cos \alpha\right) u_{2}}{2|K \cap C| \sin \alpha},
$$

verifies $\left\langle x, y_{0}\right\rangle \leq 1$ for all $x \in K \cap C$. In order to continue the argument we need to establish that $y_{0} \in K^{\circ} \cap C$. Since $\lambda_{j} \cos \alpha \leq \lambda_{i}$ for $i \neq j \in\{1,2\}$, we already know that $y_{0} \in C$. 
Next let us check that $y_{0} \in K^{\circ}$. Since $\sup _{x \in K \cap C}\left\langle x, y_{0}\right\rangle \leq 1$, we are done if we prove that for all $y \in C$,

$$
\sup _{x \in K \backslash C}\langle x, y\rangle \leq \sup _{x \in K \cap C}\langle x, y\rangle .
$$

Since $K \subset\left\{x ; \max _{i=1,2}\left\langle x, u_{i}\right\rangle \leq \lambda_{i}\right\}$, we have

$$
K \backslash C \subset\left\{x ; x \notin C, \max _{i=1,2}\left\langle x, u_{i}\right\rangle \leq \lambda_{i}\right\}=C^{\circ} \cup S_{1} \cup S_{2},
$$

where $C^{\circ}=\{x ; \forall y \in C,\langle x, y\rangle \leq 0\}$ is the dual cone of $C$ and $S_{1}$ and $S_{2}$ are two half-slabs defined by

$$
S_{i}=\left\{x ;\left\langle x, u_{i}\right\rangle \in\left[0, \lambda_{i}\right] \text { and }\left\langle x, v_{i}\right\rangle \leq 0\right\}=\left\{\lambda u_{i}-\mu v_{i} ; \lambda \in\left[0, \lambda_{i}\right], \mu \geq 0\right\} .
$$

Let $y \in C$ be fixed. Then $\left\langle v_{i}, y\right\rangle \geq 0$ hence $\sup _{x \in S_{i}}\langle x, y\rangle \leq \max \left(0,\left\langle\lambda_{i} u_{i}, y\right\rangle\right)$. Thus we deduce that

$$
\sup _{x \in K \backslash C}\langle x, y\rangle \leq \max \left(0,\left\langle\lambda_{1} u_{1}, y\right\rangle,\left\langle\lambda_{2} u_{2}, y\right\rangle\right)=\sup \left\{\langle x, y\rangle ; x \in \operatorname{conv}\left(0, \lambda_{1} u_{1}, \lambda_{2} u_{2}\right)\right\} \leq \sup _{x \in K \cap C}\langle x, y\rangle .
$$

Therefore $y_{0} \in K^{\circ} \cap C$ and we can reproduce the above reasoning to deduce

$$
\left|K^{\circ} \cap C\right| \geq \sum_{i=1}^{2}\left|\operatorname{conv}\left(y_{0}, K^{\circ} \cap C_{i}\right)\right|=\sum_{i=1}^{2} \frac{\left|K^{\circ} \cap C_{i}\right|\left\langle y_{0}, v_{i}\right\rangle}{2}=\frac{1}{2}\left\langle y_{0}, \frac{v_{1}}{\lambda_{1}}+\frac{v_{2}}{\lambda_{2}}\right\rangle .
$$

Using the definition of $y_{0}$ and $\left\langle v_{1}, v_{2}\right\rangle=-\cos \alpha$, the previous inequality gives

$$
4|K \cap C|\left|K^{\circ} \cap C\right| \geq 2-\cos \alpha\left(\frac{\lambda_{1}}{\lambda_{2}}+\frac{\lambda_{2}}{\lambda_{1}}\right) .
$$

This proves the inequality. Now let us establish the equality case. If there is equality then

$$
K^{\circ} \cap C=\operatorname{conv}\left(y_{0}, K^{\circ} \cap C_{1}, K^{\circ} \cap C_{2}\right)=\operatorname{conv}\left(0, y_{0}, \frac{u_{1}}{\lambda_{1}}, \frac{u_{2}}{\lambda_{2}}\right) \quad \text { where } \quad y_{0}:=\frac{\lambda_{1} v_{1}+\lambda_{2} v_{2}}{2|K \cap C|} .
$$

Defining the sets $T=\operatorname{conv}\left(0, u_{1} / \lambda_{1}, u_{2} / \lambda_{2}\right)$ and $Q=\left\{y \in C ;\left\langle\lambda_{i} u_{i}, y\right\rangle \leq 1, i=1,2\right\}$ and using the hypothesis, we have $T \subset K^{\circ} \cap C=\operatorname{conv}\left(T, y_{0}\right) \subset Q$. Our aim is to prove that $K^{\circ} \cap C$ is either equal to $T$ or $Q$. Since, as noticed at the beginning of the proof, our hypothesis is symmetric between $K$ and $K^{\circ}$, we can exchange their role and we get that

$$
K \cap C=\operatorname{conv}\left(0, x_{0}, \lambda_{1} u_{1}, \lambda_{2} u_{2}\right), \quad \text { where } \quad x_{0}=\frac{1}{2\left|K^{\circ} \cap C\right|}\left(\frac{v_{1}}{\lambda_{1}}+\frac{v_{2}}{\lambda_{2}}\right) .
$$

Since for every $y \in C, \sup _{x \in K}\langle x, y\rangle=\sup _{x \in K \cap C}\langle x, y\rangle$, it follows that

$$
K^{\circ} \cap C=\left\{y \in C ;\left\langle x_{0}, y\right\rangle \leq 1,\left\langle\lambda_{i} u_{i}, y\right\rangle \leq 1, i=1,2\right\}=Q \cap\left\{y ;\left\langle x_{0}, y\right\rangle \leq 1\right\} .
$$

Therefore $K^{\circ} \cap C$ doesn't have any vertex in the interior of $Q$. This implies that $y_{0}$ is in $T$ or for some $i \in\{1,2\},\left\langle\lambda_{i} u_{i}, y_{0}\right\rangle=1$. In the first case, we get $K^{\circ} \cap C=T$, in the second case, using the definition of $y_{0}$, we get $|K \cap C|=\frac{\lambda_{1} \lambda_{2} \sin \alpha}{2}=\left|\operatorname{conv}\left(0, \lambda_{1} u_{1}, \lambda_{2} u_{2}\right)\right|$, hence $K \cap C=\operatorname{conv}\left(0, \lambda_{1} u_{1}, \lambda_{2} u_{2}\right)$.

Corollary 3. Under the hypotheses of the previous proposition the inequality

$$
|K \cap C|_{2}\left|K^{\circ} \cap C\right|_{2} \geq \frac{1}{2}(1-\cos \alpha)
$$

is valid provided $\alpha \in[\pi / 2, \pi)$ or $\left|K \cap C_{1}\right|_{1}=\left|K \cap C_{2}\right|_{1}$. Moreover there is equality if and only if $K \cap C=$ $\lambda \operatorname{conv}\left(0, u_{1}, u_{2}\right)$ or $K \cap C=\left\{x \in C ;\left\langle x, \lambda u_{i}\right\rangle \leq 1, i=1,2\right\}$, for some $\lambda>0$. 
Proof. If $\alpha \in[\pi / 2, \pi)$ then $-\cos \alpha \geq 0$ and we may apply Proposition 2 and the arithmetic mean-geometric mean inequality $\left(\frac{\left|K \cap C_{1}\right|}{\left|K \cap C_{2}\right|}+\frac{\left|K \cap C_{2}\right|}{\left|K \cap C_{1}\right|}\right) / 2 \geq 1$. The equality case follows immediately.

Let us set $w(\theta)=(\cos \theta, \sin \theta)$. For $m \geq 3$ we consider the regular polygon with $m$ vertices and unit circumcircle:

$$
\mathcal{C}_{m}:=\operatorname{conv}\left\{w\left(\frac{2 k \pi}{m}\right) ; k=0, \ldots, m-1\right\} .
$$

Note that

$$
\mathcal{C}_{m}^{\circ}=\left\{x ;\left\langle x, w\left(\frac{2 k \pi}{m}\right)\right\rangle \leq 1 ; k=0, \ldots, m-1\right\}
$$

is also a regular polytope (obtained by rotating and dilating $\mathcal{C}_{m}$ ).

Proposition 4. Let $K$ be a convex subset of $\mathbb{R}^{2}$ such that $\mathcal{C}_{m} \subset K \subset \mathcal{C}_{m}^{\circ}$, then

$$
|K|\left|K^{\circ}\right| \geq\left|\mathcal{C}_{m}\right|\left|\mathcal{C}_{m}^{\circ}\right|=\mathcal{P}\left(\mathcal{C}_{m}\right),
$$

with equality if and only if $K$ is $\mathcal{C}_{m}$ or $\mathcal{C}_{m}^{\circ}$.

Proof. For $L=\mathcal{C}_{m}$ or $L=\mathcal{C}_{m}^{\circ}$, note that

$$
L \cap \mathbb{R}_{+} w\left(\frac{2 k \pi}{m}\right)=P_{\mathbb{R} w\left(\frac{2 k \pi}{m}\right)}(L) \cap \mathbb{R}_{+} w\left(\frac{2 k \pi}{m}\right)=\left[0, w\left(\frac{2 k \pi}{m}\right)\right],
$$

is valid. Hence it is inherited by $K$. For each $k=0, \ldots, m-1$ we consider the cone

$$
C(k):=\operatorname{pos}\left(w\left(\frac{2 k \pi}{m}\right), w\left(\frac{2(k+1) \pi}{m}\right)\right)
$$

with angle $\alpha=2 \pi / m$. In view of the previous remark and since the two sections of $K$ with the sides of the cone have the same measure, we may apply the later corollary to get:

$$
|K \cap C(k)|\left|K^{\circ} \cap C(k)\right| \geq \frac{1}{2}(1-\cos \alpha)
$$

with equality if and only if $K \cap C(k)$ is $\mathcal{C}_{m} \cap C(k)$ or $\mathcal{C}_{m}^{\circ} \cap C(k)$. Next, applying the arithmetic mean-geometric mean inequality

$$
\begin{aligned}
|K|\left|K^{\circ}\right| & =\left(\sum_{k=0}^{m-1}|K \cap C(k)|\right)\left(\sum_{k=0}^{m-1}\left|K^{\circ} \cap C(k)\right|\right) \\
& \geq m^{2}\left(\prod_{k=0}^{m-1}|K \cap C(k)|\left|K^{\circ} \cap C(k)\right|\right)^{\frac{1}{m}} \\
& \geq m^{2} \frac{1-\cos \alpha}{2}=\mathcal{P}\left(\mathcal{C}_{m}\right) .
\end{aligned}
$$

And if there is equality then $|K \cap C(k)|$ is independent of $k$. Therefore $K$ is $\mathcal{C}_{m}$ or $\mathcal{C}_{m}^{\circ}$.

As a consequence, we obtain a sharp lower bound on the volume product of planar convex sets with a rotation invariance.

Corollary 5. Let $K$ be a convex subset of $\mathbb{R}^{2}$ which is invariant by the linear rotation of angle $\frac{2 \pi}{m}$, where $m \in \mathbb{N}$ is at least 3 . Then

$$
\mathcal{P}(K) \geq \mathcal{P}\left(\mathcal{C}_{m}\right)
$$

with equality if and only if $K=\lambda U \mathcal{C}_{m}$, for some $\lambda>0$ and some isometry $U \in \mathcal{O}\left(\mathbb{R}^{2}\right)$. 
Proof. ¿From the rotation invariance $\mathcal{P}(K)=|K|\left|K^{\circ}\right|$. Without loss of generality we may assume that $w(0)$ is the point with maximal norm in $K$. Hence $[0, w(0)] \subset K \subset\{x ;\langle x, w(0)\rangle \leq 1\}$, by rotation invariance, this is true if we replace $w(0)$ by $w(2 k \pi / m)$. Hence $\mathcal{C}_{m} \subset K \subset \mathcal{C}_{m}^{\circ}$. We conclude with the previous proposition.

Remark 1. The above statement is of interest when $m \geq 5$ only. Indeed in dimension 2 , it is known from Mahler [13] that the triangle $\mathcal{C}_{3}$ has minimal volume product, whereas the square $\mathcal{C}_{4}$ has minimal volume product among sets having a center of symmetry.

Remark 2. We collect here useful information for further use. First note that for all integer $m \geq 3$, the regular polygon $\mathcal{C}_{m}$ is invariant by the reflection with respect to the $x$-axis. Note that $\mathcal{C}_{m}=-\mathcal{C}_{m}$ if and only if $m$ is even. Hence if $m$ is even, $\mathcal{C}_{m}$ is invariant also by the reflection with respect to the $y$-axis; it is therefore unconditional. Eventually we observe that $m \mapsto \mathcal{P}\left(\mathcal{C}_{m}\right)$ is an increasing function. Indeed, the above formula gives

$$
\mathcal{P}\left(\mathcal{C}_{m}\right)=m^{2} \frac{1-\cos (2 \pi / m)}{2}=(m \sin (\pi / m))^{2}
$$

and the function $x \mapsto \sin (x) / x$ is positive and decreasing on $[0, \pi)$.

Remark 3. After we had finished this work, we learned from Meyer that he and Böröczky, Makai and Reisner have some similar results for the volume product of planar convex bodies in a paper in progress.

\section{Preliminaries in arbitrary dimension}

It is natural to ask about bodies of minimal volume product under various invariance assumptions, in higher dimensions. An important technical step in the previous section was to divide the convex set $K$ under study according to a collection of convex cones having the property that for all $y \in C, \sup _{x \in K \cap C}\langle x, y\rangle=$ $\sup _{x \in K}\langle x, y\rangle$. For the sake of shortness we will sometimes say that $C$ has the norming property for $K$. The goal of this section is to provide such good cones when $K$ has a large group of isometries. Our argument is inspired by the elementary observation that when $K$ is invariant by a hyperplane symmetry $S_{H}$ then $K \cap H=P_{H}(K)$. Nevertheless more work is needed as we will have to consider cones with empty interior. Our main result in this section is the following proposition.

Proposition 6. Let $G$ be a finite subgroup of $\mathcal{O}\left(\mathbb{R}^{n}\right)$. Let $v_{1}, \ldots, v_{m}$ be $m$ unit vectors in $\mathbb{R}^{n}$ such that $S_{v_{i}^{\perp}} \in G$ for all $i=1, \ldots, m$. Assume that the cone

$$
D:=\bigcap_{i=1}^{m}\left\{x \in \mathbb{R}^{n} ;\left\langle x, v_{i}\right\rangle \geq 0\right\}
$$

is $n$-dimensional. Let $K \subset \mathbb{R}^{n}$ be a $G$-invariant convex body containing the origin. If $C$ is a facial cone of $D$ or if $C=D$ then $P_{C} K=K \cap C$ and $C$ has the norming property for $K$ : for all $y \in C$,

$$
\sup _{x \in K \cap C}\langle x, y\rangle=\sup _{x \in K}\langle x, y\rangle \text {. }
$$

In order to prove Proposition 6 we first establish some lemmata. The following basic fact gives a sufficient condition for ensuring the norming property of general cones.

Lemma 7. Let $C$ be a closed convex cone and $K$ be a convex set. Let $P_{C}$ denote the projection onto $C$. If $P_{C} K \subset K$ (or equivalently $P_{C} K=K \cap C$ ) then for all $y \in C$,

$$
\sup _{x \in K}\langle x, y\rangle=\sup _{x \in K \cap C}\langle x, y\rangle .
$$


Proof. Recall that $P_{C} x$ is the unique closest point to $x$ in $C$. Classically using convexity, the closest point property gives for $y$ in $C$ and $t \in(0,1)$,

$$
\left|x-P_{C} x\right|^{2} \leq\left|x-\left((1-t) P_{C} x+t y\right)\right|^{2} .
$$

Expanding and letting $t$ to zero gives the characteristic property $\left\langle x-P_{C} x, y-P_{C} x\right\rangle \leq 0$. Since $C$ is a cone, this has to be satisfied when $y$ is replaced by $t y$ for any $t>0$. This implies that $\left\langle x-P_{C} x, y\right\rangle \leq 0$ or

$$
\langle x, y\rangle \leq\left\langle P_{C} x, y\right\rangle .
$$

Hence if $x \in K$, our hypothesis ensures that $P_{C} x \in K$. Hence for all $y \in C$, we have found a point in $C$ with a bigger scalar product with $y$ than $x$.

We note in the next lemma that the property $P_{C} K \subset K$ can be guaranteed by the stability of $K$ under orthogonal projections onto the vector spaces appearing in the facial structure of $C$.

Lemma 8. Let $C$ be a convex cone of dimension $d$, in $\mathbb{R}^{n}$, with $2 \leq d \leq n$, and $K$ be a convex set containing the origin. Let $\mathcal{E}$ be the family of vector spaces spanned by all the faces of $C$ (of dimension 1 to $d-1$ ). If for all $E \in \mathcal{E}$, we have $P_{E} K=K \cap E$ then $P_{C} K=K \cap C$.

Proof. Let $x \in K$. If $x \in C$ we have nothing to prove. So assume $x \notin C$. Hence $P_{C} x \in \partial C$. If $P_{C} x=0$ we are done. If $P_{C} x \neq 0$, let $F$ be the $k$-face of $C$ containing $P_{C} x$ and of minimal dimension $k \geq 1$. Let $E:=\operatorname{span}(F)$. By minimality of $F, P_{C} x$ belongs to the relative interior of $F$ (with respect to $E$ ). Hence the closest point property of $P_{C} x$ implies actually that $x-P_{C} x$ is orthogonal to all the directions in $E$. This means that $P_{C} x=P_{E} x$. Since we have assumed that $P_{E} K \subset K$, we conclude that $P_{C} x \in K$.

The next statement allows to verify the hypotheses of the latter lemma from properties of the isometry group of a convex body. Given a group $G$ acting on $\mathbb{R}^{n}$, recall that $\operatorname{Fix}(G)=\left\{x \in \mathbb{R}^{n} ; \forall g \in G, g x=x\right\}$.

Lemma 9. Let $E$ be a subspace of $\mathbb{R}^{n}$. Let $G$ be a finite subgroup of the orthogonal group $O\left(\mathbb{R}^{n}\right)$ such that

$$
\operatorname{Fix}\left(\left\{g \in G ; g_{\mid E}=I d_{E}\right\}\right)=E .
$$

Then any $G$-invariant convex set $K \subset \mathbb{R}^{n}$ satisfies $P_{E} K=K \cap E$.

Proof. Start from $x \in K$. Our goal is to show that $P_{E} x$ is in $K$. Let $G^{\prime}$ be the subgroup of $G$ composed of the isometries which leave $E$ pointwise invariant. Let $h:=\frac{1}{\operatorname{card}\left(G^{\prime}\right)} \sum_{g \in G^{\prime}} g$. Since $x \in K$ and $K$ is invariant by $G, g x \in K$ hence by convexity

$$
h x=\frac{1}{\operatorname{card}\left(G^{\prime}\right)} \sum_{g \in G^{\prime}} g x \in K .
$$

We are done if $h=P_{E}$. This is what we check next.

By definition it is obvious that $h_{\mid E}=\operatorname{Id}_{E}$. Also any isometry $g \in G^{\prime}$ satisfies $g_{\mid E}=\operatorname{Id}_{E}$ hence $g\left(E^{\perp}\right) \subset$ $E^{\perp}$ and consequently $h\left(E^{\perp}\right) \subset E^{\perp}$. In order to prove that $h=P_{E}$ it suffices to show that $\operatorname{Im}(h) \subset E$. Let $y \in \operatorname{Im}(h)$ and $u$ such that $y=h(u)$. Then by the group property, for any $g_{0} \in G^{\prime}$

$$
g_{0} y=g_{0} h(u)=\frac{1}{\operatorname{card}\left(G^{\prime}\right)} \sum_{g \in G^{\prime}} g_{0} g(u)=\frac{1}{\operatorname{card}\left(G^{\prime}\right)} \sum_{g^{\prime} \in G^{\prime}} g^{\prime}(u)=h(u)=y .
$$

Hence $y \in \operatorname{Fix}\left(G^{\prime}\right)$. Our hypothesis yields $y \in E$ as required. 
Proof of Proposition 6. In view of Lemmata 7, 8 and 9, it is enough to show that for all facial cones $C$ of $D$ (of any dimension $1 \leq d \leq n-1$ ), the space $E:=\operatorname{span}(C)$ satisfies that

$$
\operatorname{Fix}\left(\left\{g \in G ; g_{\mid E}=\operatorname{Id}_{E}\right\}\right) \subset E .
$$

Since $C$ is a $d$-dimensional face of $D$, it is the intersection of $D$ with $n-d$ hyperplanes taken among the family $\left(\left\{x \in \mathbb{R}^{n} ;\left\langle x, v_{i}\right\rangle=0\right\}\right)_{i=1}^{m}$. It follows that there exists a subset $I_{E}$ of $\{1, \ldots, m\}$ of cardinality $n-d$ such that

$$
E=\bigcap_{i \in I_{E}}\left\{x \in \mathbb{R}^{n} ;\left\langle x, v_{i}\right\rangle=0\right\} .
$$

Consequently, for all $i \in I_{E}, S_{v_{i}^{\perp}}$ leaves $E$ pointwise invariant. Hence

$$
\operatorname{Fix}\left(\left\{g \in G ; g_{\mid E}=\operatorname{Id}_{E}\right\}\right) \subset \operatorname{Fix}\left(\left\{S_{v_{i}^{\perp}} ; i \in I_{E}\right\}\right)=\bigcap_{i \in I_{E}} v_{i}^{\perp}=E,
$$

as required.

Remark 4. In the results of this section, the finiteness assumption on the groups $G$ could be replaced by compactness. We would average according to the Haar probability measure instead of the counting measure.

\section{Regular polytopes}

Corollary 5 about regular polygons suggests the following question: if $K$ has the same symmetries as a regular convex polytope, does it have a bigger volume product? The aim of this section is to show that it is indeed the case:

Theorem 10. Let $P$ be a regular polytope in $\mathbb{R}^{n}$ centered at the origin and let $\mathcal{O}(P)$ be its isometry group. Let $K$ be an $\mathcal{O}(P)$-invariant convex body in $\mathbb{R}^{n}$. Then

$$
\mathcal{P}(K)=|K|\left|K^{\circ}\right| \geq|P|\left|P^{\circ}\right|=\mathcal{P}(P),
$$

with equality if and only if $K$ is a dilate of $P$ or a dilate of $P^{\circ}$.

Let us first recall what is a regular polytope (see [3], [4] and [9]). To do this, we first need the definition of a flag. A flag of a polytope $P$ in $\mathbb{R}^{n}$ is an $n$-tuple $\mathcal{F}=\left(F_{0}, F_{1}, \ldots, F_{n-1}\right)$, where $F_{0}$ is a vertex of $P, F_{1}$ is an edge of $P$ containing $F_{0}$, and for all $k \in\{1, \ldots, n-1\} F_{k}$ is a $k$-dimensional face of $P$ containing $F_{k-1}$. A polytope $P$ in $\mathbb{R}^{n}$ of dimension $n$ is regular if for every two flags $\mathcal{F}$ and $\mathcal{F}^{\prime}$ of $P$ there exists an element $g \in \mathcal{O}(P)$ such that $g(\mathcal{F})=\mathcal{F}^{\prime}$.

From this definition, one may deduce a few useful facts: for every $0 \leq k \leq n-1$, the $k$-faces of a regular polytope are regular and isometric. Since the center of gravity of $P$ is fixed by $\mathcal{O}(P)$, every vertices of $P$ are on the same sphere centered at the center of gravity. Notice that any linear isometry $g$ of $\mathcal{O}\left(\mathbb{R}^{n}\right)$ is uniquely determined by its image on a flag not containing the origin. Moreover, for any two adjacent facets $F$ and $F^{\prime}$ of $P$, i.e. such that $F \cap F^{\prime}$ is a $(n-2)$-face, consider the flags $\mathcal{F}=\left(F_{0}, F_{1}, \ldots, F_{n-3}, F \cap F^{\prime}, F\right)$ and $\mathcal{F}^{\prime}=\left(F_{0}, F_{1}, \ldots, F_{n-3}, F \cap F^{\prime}, F^{\prime}\right)$, where $\left(F_{0}, F_{1}, \ldots, F_{n-3}\right)$ is a flag of $F \cap F^{\prime}$. Then there is $g \in \mathcal{O}(P)$ such that $g(\mathcal{F})=\mathcal{F}^{\prime}$. Thus $H:=\operatorname{span}\left(F \cap F^{\prime}\right)$ is fixed by $g$, therefore $g$ is the reflection $\sigma_{H}$ with respect to the hyperplane $H$ and one has $\sigma_{H}(F)=F^{\prime}$.

Let us discuss the interest of Theorem 10. Notice that the property of beeing a regular polytope is in fact very restrictive. In dimension 2 , there are infinitely many regular polygons, as explained in section 2 , but in all dimension $n \geq 3$, the list is rather short: five regular polytopes in dimension 3, six regular polytopes in dimension 4 and only three regular polytopes in dimension $n \geq 5$ as was shown by the classification of regular polytopes established by Coxeter [3]. Moreover, the case of the cube and cross-polytope, dual of each other, follows from the reverse Santaló inequality for unconditional bodies of Saint Raymond [23], which 
actually have less symmetries. Thus the interest of Theorem 10 lies in the remaining cases: the simplex in all dimensions $n \geq 3$, the dodecahedron and the icosahedron (dual of each other) in dimension 3 and the 24-cell $\operatorname{conv}\left(B_{1}^{4}, \frac{1}{2} B_{\infty}^{4}\right)$, the 120-cell and 600-cell in 4 dimensions (the latter two being also dual of each other). We shall treat these cases altogether without using the classification of regular polytopes.

The strategy will be to divide $K$ according to the cones generated by the facets of $P$ and to adapt Meyer's argument of [14] in this context. This will naturally lead to study the intersection of $K$ and its polar with the cones generated by all $k$-faces of $P$, for all $k$. The following lemma gathers the geometric conditions that allow to estimate the volume product in a $k$-dimensional cone, in terms of the volume products in its facets when the situation is symmetric enough. This the heart of the argument of [14].

Lemma 11. Let $K$ be a convex body in $\mathbb{R}^{n}$ with $0 \in K$. Let $1 \leq k \leq n-1$, C be a ( $\left.k+1\right)$-dimensional polyhedral convex cone in $\mathbb{R}^{n}$ and $C_{1}, \ldots, C_{m}$ be the $k$-faces of $C$. For $1 \leq i \leq m$, let $v_{i} \in \operatorname{span}(C)$ be the unit vector normal to $C_{i}$ in such a way that $\left\langle x, v_{i}\right\rangle \geq 0, \forall x \in C$. Let us assume that

1. The quantities $\left|K \cap C_{i}\right|_{k}$ and $\left|K^{\circ} \cap C_{i}\right|_{k}$ do not depend on the value of $i$.

2. The vector $w:=\sum_{i=1}^{m} v_{i}$ lies in $C$.

3. For all $y \in C, \sup _{x \in K}\langle x, y\rangle=\sup _{x \in K \cap C}\langle x, y\rangle$.

Let $x_{0}=\frac{\left|K^{\circ} \cap C_{1}\right|_{k}}{(k+1)\left|K^{\circ} \cap C\right|_{k+1}} w$ and $y_{0}=\frac{\left|K \cap C_{1}\right|_{k}}{(k+1)|K \cap C|_{k+1}} w$. Then $y_{0} \in K^{\circ} \cap C$ and

$$
|K \cap C|_{k+1}\left|K^{\circ} \cap C\right|_{k+1} \geq\left|K \cap C_{1}\right|_{k}\left|K^{\circ} \cap C_{1}\right|_{k} \frac{|w|^{2}}{(k+1)^{2}},
$$

with equality in (1) if and only if $K^{\circ} \cap C=\bigcup_{i=1}^{m} \operatorname{conv}\left(y_{0}, K^{\circ} \cap C_{i}\right)$. Moreover any $y \in K^{\circ} \cap C$, satisfies $\left\langle y, x_{0}\right\rangle \leq 1$ with equality if and only if $K^{\circ} \cap C=\bigcup_{i=1}^{m} \operatorname{conv}\left(y, K^{\circ} \cap C_{i}\right)$.

Proof. For any $x \in K \cap C$, we have

$$
|K \cap C|_{k+1} \geq \sum_{i=1}^{m}\left|\operatorname{conv}\left(x, K \cap C_{i}\right)\right|_{k+1}=\sum_{i=1}^{m} \frac{\left|K \cap C_{i}\right|_{k}\left\langle x, v_{i}\right\rangle}{k+1}=\frac{\left|K \cap C_{1}\right|_{k}}{k+1}\langle x, w\rangle,
$$

where $w=\sum_{i=1}^{m} v_{i}$. This means that $\left\langle x, y_{0}\right\rangle \leq 1$ for all $x \in K \cap C$. Since $y_{0} \in C$ it follows that $\sup _{z \in K}\left\langle z, y_{0}\right\rangle=\sup _{x \in K \cap C}\left\langle x, y_{0}\right\rangle \leq 1$. This allows us to conclude that $y_{0} \in K^{\circ} \cap C$. Hence we may apply the preceding dissection argument for $K^{\circ}$ and the point $y_{0}$. This leads to

$$
\left|K^{\circ} \cap C\right|_{k+1} \geq \sum_{i=1}^{m}\left|\operatorname{conv}\left(y_{0}, K^{\circ} \cap C_{i}\right)\right|_{k+1}=\sum_{i=1}^{m} \frac{\left|K^{\circ} \cap C_{i}\right|_{k}\left\langle y_{0}, v_{i}\right\rangle}{k+1}=\frac{\left|K^{\circ} \cap C_{1}\right|_{k}}{k+1}\left\langle y_{0}, w\right\rangle
$$

Substituting the expression of $y_{0}$ yields

$$
|K \cap C|_{k+1}\left|K^{\circ} \cap C\right|_{k+1} \geq\left|K \cap C_{1}\right|_{k}\left|K^{\circ} \cap C_{1}\right|_{k} \frac{|w|^{2}}{(k+1)^{2}},
$$

with equality if and only if $K^{\circ} \cap C=\bigcup_{i=1}^{m} \operatorname{conv}\left(y_{0}, K^{\circ} \cap C_{i}\right)$. Applying the above argument to any $y \in K^{\circ} \cap C$ instead of $y_{0}$, we get that $\left\langle y, x_{0}\right\rangle \leq 1$ with equality if and only if $K^{\circ} \cap C=\bigcup_{i=1}^{m} \operatorname{conv}\left(y, K^{\circ} \cap C_{i}\right)$.

Next we show how this applies to bodies with the symmetries of a regular polytope. 
Lemma 12. Let $P \subset \mathbb{R}^{n}$ be a regular polytope with barycenter at the origin and let $K \subset \mathbb{R}^{n}$ be an $\mathcal{O}(P)$ invariant convex body. Let $0 \leq k \leq n-1, F$ be a $k$-face of $P$ and $C=\operatorname{pos}(F)$.

If $k \neq 0$, let $C_{1}, \ldots, C_{m}$ be the $k$-faces of $C$. Let $v_{i}$ be the unit normal vector to $\operatorname{span}\left(\mathrm{C}_{\mathrm{i}}\right)($ within $\operatorname{span}(C))$ pointing in the direction of $C$. Then

$$
|K \cap C|_{k+1}\left|K^{\circ} \cap C\right|_{k+1} \geq\left|K \cap C_{1}\right|_{k}\left|K^{\circ} \cap C_{1}\right|_{k} \frac{\left|\sum_{i} v_{i}\right|^{2}}{(k+1)^{2}}
$$

and there is equality if and only if $K \cap C$ is homothetic to $P \cap C$ or $P^{\circ} \cap C$.

If $k=0$ then $|K \cap C|_{1}\left|K^{\circ} \cap C\right|_{1}=1$.

Proof. The proof being a bit long we shall divide it in three steps.

Step 1: the inequality. Notice first that since $P$ is regular, for any $0 \leq \ell \leq n-1$, any two $\ell$-faces of $P$ are isometric by an element of $\mathcal{O}(P)$ and so are the cones $D_{1}$ and $D_{2}$ that they generate. Since $K$ is $\mathcal{O}(P)$-invariant, $K \cap D_{1}$ and $K \cap D_{2}$ are isometric, in particular they have the same $(\ell+1)$-volume. Moreover for every $g \in \mathcal{O}\left(\mathbb{R}^{n}\right)$, if $K=g K$ then by polarity $K^{\circ}=(g K)^{\circ}=\left(g^{*}\right)^{-1} K^{\circ}=g K^{\circ}$. Hence, $K$ and $K^{\circ}$ have the same isometry group. Therefore the same is true for $K^{\circ}$.

Applying Proposition 6 for $G=\mathcal{O}(P)$ and for the cone generated by a facet of $P$, we deduce that for any cone $D$ generated by any face of $P$ one has $P_{D}(K)=K \cap D$ and $D$ has the norming property for $K$ : for all $y \in D, \sup _{x \in K}\langle x, y\rangle=\sup _{x \in K \cap D}\langle x, y\rangle$.

First, we treat the case $k=0: F$ is a vertex $u$ of $P$ and $C=\operatorname{pos}(F)=\mathbb{R}^{+} u$. Hence there is $\lambda>0$ such that $K \cap C=[0, \lambda u]$. We choose the normalization of $P$ and we dilate $K$ so that $\|u\|_{2}=\lambda=1$. In this case, as in the planar case, the property $P_{C}(K)=K \cap C$ is equivalent to $[0, u] \subset K \subset\{x ;\langle x, u\rangle \leq 1\}$. And as before, this property passes to the polar $[0, u] \subset K^{\circ} \subset\{x ;\langle x, u\rangle \leq 1\}$. Hence $K^{\circ} \cap C=[0, u]$, taking volumes, we get $|K \cap C|\left|K^{\circ} \cap C\right|=1$.

Now we assume that $k \geq 1$. The proof of the inequality consists in applying the preceding lemma. We shall verify the hypotheses $1,2,3$. It follows from the beginning of the proof that the first and the third hypotheses are satisfied.

To verify the second hypothesis, denote by $F_{1}, \ldots, F_{m}$ the $(k-1)$-faces of $F$ which compose the relative boundary of $F$ and notice that $C_{i}=\operatorname{pos}\left(F_{i}\right)$. Observe that one can find $g_{i} \in \mathcal{O}(P)$ such that $g_{i}\left(F_{1}\right)=F_{i}$ and also $g_{i}(F)=F$. To see this, one simply builds two flags of $P$ by extending the "short flags" $\left(F_{1}, F\right)$ and $\left(F_{i}, F\right)$. Since $P$ is regular there exists $g_{i} \in \mathcal{O}(P)$ which maps one flag to the other. In particular $g_{i}\left(F_{1}\right)=F_{i}, g_{i}(F)=F$ and consequently $g_{i}\left(C_{1}\right)=C_{i}, g_{i}(C)=C$. Since $g_{i}$ preserves orthogonality, it is plain that $v_{i}=g_{i}\left(v_{1}\right)$. As $g_{i}(F)=F, g_{i}$ permutes the $(k-1)$-faces $\left\{F_{1}, \ldots, F_{m}\right\}$. It also acts as a bijection on the set of their normal vectors $\left\{v_{1}, \ldots, v_{m}\right\}$. Since $w=\sum_{j} v_{j}$, one concludes that $g_{i}(w)=w$, hence

$$
\left\langle w, v_{1}\right\rangle=\left\langle g_{i}(w), g_{i}\left(v_{1}\right)\right\rangle=\left\langle w, v_{i}\right\rangle
$$

Summing over $i$, we get

$$
m\left\langle w, v_{1}\right\rangle=\sum_{i=1}^{m}\left\langle w, v_{i}\right\rangle=|w|^{2} \geq 0
$$

Hence for all $i \in\{1, \ldots, m\},\left\langle w, v_{i}\right\rangle \geq 0$ and we may conclude that $w \in C$. In fact, one gets more: each of the isometries $g_{1}, \ldots g_{m}$ leaves $F$ globally invariant and fixes $w$. Moreover they can have only one common fixed point in $F$, hence $w$ is a vector which is normal to $F$ and if we denote by $d$ the distance between $F$ and the origin, then the center of gravity of $F$ is $d w /|w|$.

All the hypotheses of Lemma 11 have been checked so its conclusion is available:

$$
|K \cap C|_{k+1}\left|K^{\circ} \cap C\right|_{k+1} \geq\left|K \cap C_{1}\right|_{k}\left|K^{\circ} \cap C_{1}\right|_{k} \frac{|w|^{2}}{(k+1)^{2}},
$$


with equality if and only if $K^{\circ} \cap C=\bigcup_{i=1}^{m} \operatorname{conv}\left(y_{0}, K^{\circ} \cap C_{i}\right)$, where $y_{0}=\frac{\left|K \cap C_{1}\right|_{k}}{(k+1)|K \cap C|_{k+1}} w$.

Step 2: there is equality for $K=P$. We only need to check that $P^{\circ} \cap C=\bigcup_{i=1}^{m} \operatorname{conv}\left(y_{0}, P^{\circ} \cap C_{i}\right)$, where $y_{0}=\frac{\left|P \cap C_{1}\right|_{k}}{(k+1)|P \cap C|_{k+1}} w$. Firstly, we express $y_{0}$ in a more geometric way. We already saw that $d \frac{w}{|w|}$ is the center of the face $F$ of $P$. Since $C=\operatorname{pos}(F)$, we have $P \cap C=\bigcup_{i=1}^{m} \operatorname{conv}\left(\frac{d w}{|w|}, P \cap C_{i}\right)$, hence taking volumes

$$
\begin{aligned}
|P \cap C|_{k+1} & =\sum_{i=1}^{m}\left|\operatorname{conv}\left(\frac{d w}{|w|}, P \cap C_{i}\right)\right|_{k+1}=\sum_{i=1}^{m} \frac{\left|P \cap C_{i}\right|_{k}}{k+1}\left\langle\frac{d w}{|w|}, v_{i}\right\rangle \\
& =\frac{d}{k+1}\left|P \cap C_{1}\right|_{k}|w|,
\end{aligned}
$$

using that $\left|P \cap C_{i}\right|_{k}=\left|P \cap C_{1}\right|_{k}$ and $w=\sum_{i} v_{i}$. Consequently,

$$
y_{0}=\frac{\left|P \cap C_{1}\right|_{k}}{(k+1)|P \cap C|_{k+1}} w=\frac{1}{d} \frac{w}{|w|} .
$$

As we already mentioned, for $y \in C, \sup _{x \in P}\langle x, y\rangle=\sup _{x \in P \cap C}\langle x, y\rangle$. Denote by $u_{1}, \ldots, u_{\ell}$ the vertices of $F$ (which are the non-zero vertices of $P \cap C=[0,1] \cdot F$ ). Then the latter equality implies that for $y \in C$,

$$
y \in P^{\circ} \Longleftrightarrow \forall i \in\{1, \ldots, \ell\},\left\langle y, u_{i}\right\rangle \leq 1 \text {. }
$$

Observe that $y_{0} \in P^{\circ} \cap C$ and actually saturates the above inequalities. Indeed, given two vertices $u_{i}, u_{j}$ of $F$, there exists an isometry $g \in \mathcal{O}(P)$ such that $g(F)=F$ and $g\left(u_{i}\right)=u_{j}$ (to see this, extend the short flags $\left(\left\{u_{i}\right\}, F\right)$ and $\left(\left\{u_{j}\right\}, F\right)$ into maximal flags of $P$, which are isometric since $P$ is regular). Clearly $g(w)=w$ (since the barycenter of $F$ is preserved) and $\left\langle w, u_{j}\right\rangle=\left\langle g(w), g\left(u_{i}\right)\right\rangle=\left\langle w, u_{i}\right\rangle$. Hence

$$
\left\langle\frac{1}{d} \frac{w}{|w|}, u_{i}\right\rangle=\left\langle\frac{1}{d} \frac{w}{|w|}, \frac{\sum_{j=1}^{\ell} u_{j}}{\ell}\right\rangle=\left\langle\frac{1}{d} \frac{w}{|w|}, d \frac{w}{|w|}\right\rangle=1,
$$

where we have used that $\left(\sum_{j} u_{j}\right) / \ell$ is the barycenter of $F$, which is easy since it is invariant by all isometries of $F$.

We are now ready to check that $P^{\circ} \cap C$ is a subset of $\bigcup_{i=1}^{m} \operatorname{conv}\left(y_{0}, P^{\circ} \cap C_{i}\right)$ (the reverse inclusion is obvious since $\left.y_{0} \in P^{\circ} \cap C\right)$. Let $y \in P^{\circ} \cap C$. Consider the half-line starting at $y_{0}$ and passing through $y$

$$
L:=\left\{y_{0}+\lambda\left(y-y_{0}\right) ; \lambda \geq 0\right\} .
$$

First, we remark that $L$ is not included in $C$ : since $d \frac{w}{|w|} \in P$, we have $\left\langle y, d \frac{w}{|w|}\right\rangle \leq 1=\left\langle y_{0}, d \frac{w}{|w|}\right\rangle$. Consequently for $\lambda \geq 0,\left\langle y_{0}+\lambda\left(y-y_{0}\right), d \frac{w}{|w|}\right\rangle=1+\lambda\left\langle y-y_{0}, d \frac{w}{|w|}\right\rangle \leq 1$. Recall that $d w /|w|$ is the center of the face $F$ and is orthogonal to the direction of this face. If $L \subset C$ the latter inequality implies that $L \subset \frac{1}{d} P \cap C=[0,1 / d] \cdot F$. This is impossible as this is a bounded set. Hence the half-line $L$ has to exit from $C$ at some point $z$ of the boundary of $C$, which is $\cup_{i=1}^{m} C_{i}$ hence $z \in C_{i}$ for some $i$ and since $z \in L, z=y_{0}+\lambda\left(y-y_{0}\right)$ for some $\lambda \geq 0$ and $\lambda>0$ since $z \neq y_{0}$ because $y_{0}$ is in the interior of $C$. It follows that $\left\langle z, u_{j}\right\rangle=\left\langle(1-\lambda) y_{0}+\lambda y, u_{j}\right\rangle \leq 1$, for any $j$. Hence $z \in P^{\circ} \cap C_{i}$. Therefore

$$
y=\frac{1}{\lambda} z+\left(1-\frac{1}{\lambda}\right) y_{0} \in \operatorname{conv}\left(y_{0}, P^{\circ} \cap C_{i}\right) .
$$

Hence we have established that (2) is an equality when $K=P$.

Step 3: characterization of the equality case. Now let us prove that if $K$ satisfies equality in (2) then $K \cap C$ is a dilate of $P \cap C$ or $P^{\circ} \cap C$. We choose the same normalization as before: the vertices $u_{1}, \cdots, u_{\ell}$ of $F$ are unit vectors and for all $i,\left[0, u_{i}\right] \subset K \subset\left\{x ;\left\langle x, u_{i}\right\rangle \leq 1\right\}$. This implies that

$$
P \cap C=\operatorname{conv}(0, F) \subset K \cap C \subset\left\{x ;\left\langle x, u_{i}\right\rangle \leq 1, \forall 1 \leq i \leq \ell\right\}=P^{\circ} \cap C
$$


and the same for $K^{\circ} \cap C$. To avoid confusion let us denote by $y_{0}(P)=\frac{\left|P \cap C_{1}\right|_{k}}{(k+1)|P \cap C|_{k+1}} w=\frac{1}{d} \frac{w}{|w|}$ the extreme point of $P^{\circ}$ in $C$ such that $P^{\circ} \cap C=\bigcup_{i=1}^{m} \operatorname{conv}\left(y_{0}, P^{\circ} \cap C_{i}\right)$.

We already saw from the equality case in Lemma 11 that

$$
K^{\circ} \cap C=\bigcup_{i=1}^{m} \operatorname{conv}\left(y_{0}, K^{\circ} \cap C_{i}\right), \quad \text { where } \quad y_{0}=\frac{\left|K \cap C_{1}\right|_{k}}{(k+1)|K \cap C|_{k+1}} w .
$$

Moreover any $y \in K^{\circ} \cap C$, satisfies $\left\langle y, x_{0}\right\rangle \leq 1$ with equality if and only if $K^{\circ} \cap C=\bigcup_{i=1}^{m} \operatorname{conv}\left(y, K^{\circ} \cap C_{i}\right)$. Since our hypotheses are symmetric between $K$ and $K^{\circ}$, we can also apply Lemma 11 to $K^{\circ}$ and since there is equality we deduce that

$$
K \cap C=\bigcup_{i=1}^{m} \operatorname{conv}\left(x_{0}, K \cap C_{i}\right) \quad \text { where } \quad x_{0}=\frac{\left|K^{\circ} \cap C_{1}\right|_{k}}{(k+1)\left|K^{\circ} \cap C\right|_{k+1}} w .
$$

Moreover any $x \in K \cap C$, satisfies $\left\langle x, y_{0}\right\rangle \leq 1$ with equality if and only if $K \cap C=\bigcup_{i=1}^{m} \operatorname{conv}\left(x, K \cap C_{i}\right)$.

We distinguish two cases:

1. If $y_{0}$ is the unique point in $K^{\circ} \cap C$ such that $K^{\circ} \cap C=\bigcup_{i=1}^{m} \operatorname{conv}\left(y_{0}, K^{\circ} \cap C_{i}\right)$, i.e. such that $\left\langle x_{0}, y_{0}\right\rangle=1$. Let us prove that $K^{\circ} \cap C=P^{\circ} \cap C$. Since $K^{\circ} \cap C \subset P^{\circ} \cap C$, we only need to prove that the vertices of $P^{\circ} \cap C$ are in $K^{\circ} \cap C$.

Let $u \in\left\{u_{1}, \ldots, u_{\ell}\right\}$. Since $K \cap C=\cup_{i=1}^{m} \operatorname{conv}\left(x_{0}, K \cap C_{i}\right)$ and $u \in \partial K \cap C_{j}$ for some $j$, we deduce that the point $x:=\frac{x_{0}+u}{2}$ is in $\partial K$. Since $C$ satisfies the norming property for $K^{\circ}$, it follows that there exists $y \in K^{\circ} \cap C$ such that $\langle x, y\rangle=1$. Hence

$$
\left\langle\frac{x_{0}+u}{2}, y\right\rangle=\frac{\left\langle x_{0}, y\right\rangle+\langle u, y\rangle}{2}=1 .
$$

Since $x_{0}, u \in K$ and $y \in K^{\circ}$, one deduces that $\left\langle x_{0}, y\right\rangle=1$ and $\langle u, y\rangle=1$. But $y_{0}$ is the unique point in $K^{\circ} \cap C$ such that $\left\langle x_{0}, y_{0}\right\rangle=1$ hence $y_{0}=y$, which implies that $\left\langle u, y_{0}\right\rangle=1$. Since $u$ was an arbitrary vertex of $F$, we deduce that $\left\langle u_{i}, y_{0}\right\rangle=1$, for every $i \in\{1, \ldots, \ell\}$. Therefore $y_{0}=y_{0}(P)$. Hence the vertex $y_{0}(P)$ of $P^{\circ} \cap C$ is in $K^{\circ} \cap C$. Next, by Proposition 6 we know that for any face $D$ of $C$ (of any dimension), $P_{D}\left(K^{\circ}\right) \subset K^{\circ}$, hence $P_{D}\left(y_{0}(P)\right) \in K^{\circ} \cap C$.

To reach our goal, it is enough to verify that all the vertices of $P^{\circ} \cap C$ are the projections of $y_{0}(P)$ on all the faces (of any dimension) of $C$. We prove this inductively. Let $C_{1}$ be a facet of $C$ and let $y_{1}(P)$ be the unique vertex of $P^{\circ} \cap C_{1}$ in the relative interior of $C_{1}$. Let $I \subset\{1, \ldots, \ell\}$ such that the set of vertices of $F_{1}$ is $\left\{u_{i}, i \in I\right\}$. For $i \in I$, notice that $u_{i} \in C_{1}$, hence $\left\langle v_{1}, u_{i}\right\rangle=0$. We know that $P^{\circ} \cap C_{1}=\left\{y \in C_{1} ;\left\langle y, u_{i}\right\rangle \leq 1, \forall i \in I\right\}$ and $y_{1}(P)$ is the unique point of $C_{1}$ (and actually of $\operatorname{span}\left(C_{1}\right)$ ) such that $\left\langle y_{1}(P), u_{i}\right\rangle=1, \forall i \in I$. Consider the projection $z:=P_{\operatorname{span}\left(C_{1}\right)}\left(y_{0}(P)\right)=y_{0}(P)-\left\langle y_{0}(P), v_{1}\right\rangle v_{1}$. Since $\left\langle y_{0}(P), u_{i}\right\rangle=1$ and $\left\langle v_{1}, u_{i}\right\rangle=0$, for all $i \in I$, it follows that $\left\langle z, u_{i}\right\rangle=1, \forall i \in I$. Hence $z=y_{1}(P)$ and it belongs to $C_{1}$. Therefore $y_{1}(P)=P_{\mathrm{span}\left(C_{1}\right)}\left(y_{0}(P)\right)=P_{C_{1}}\left(y_{0}(P)\right)$. Iterating this procedure, we obtain that all the vertices of $P^{\circ} \cap C$ are the projections of $y_{0}(P)$ on the faces of $C$. Combining the above arguments, we may conclude that $K^{\circ} \cap C=P^{\circ} \cap C$.

2. If there is $y_{1} \neq y_{0}$ in $K^{\circ} \cap C$ such that $\left\langle x_{0}, y_{1}\right\rangle=1$. Let us prove that $K^{\circ} \cap C=P \cap C$. Note that $H:=\left\{y ;\left\langle x_{0}, y\right\rangle=1\right\}$ is a supporting hyperplane of $K^{\circ} \cap C$ and that $y_{0}, y_{1}$ are contact points. Using isometries of $P$ (hence of $K^{\circ}$ ) which leave $C$ globally invariant and permute its facets, we obtain new contact points $y_{2}, \ldots y_{m}$, as the images of $y_{1}$. It is readily checked that $\operatorname{conv}\left(y_{1}, \ldots, y_{m}\right)$ is a convex body in $H$ which contains $y_{0}$ in its interior. So the boundary of $K^{\circ} \cap C$ is flat in a neighborhood of $y_{0}$. However the boundary of $K^{\circ} \cap C$ is also star-shaped at $y_{0}$ since $K^{\circ} \cap C=\cup_{i} \operatorname{conv}\left(y_{0}, K^{\circ} \cap C_{i}\right)$. Combining these two facts, we deduce that $K^{\circ} \cap C=C \cap\left\{y ;\left\langle y, x_{0}\right\rangle \leq 1\right\}$. Since, by our normalization, $u_{1}, \ldots, u_{m} \in \partial K^{\circ} \cap C$ we conclude that $K^{\circ} \cap C=P \cap C$.

The proof is therefore complete. 
Notice that the inequality of the preceding lemma may be rewritten as

$$
\frac{|K \cap C|_{k+1}\left|K^{\circ} \cap C\right|_{k+1}}{\left|K \cap C_{1}\right|_{k}\left|K^{\circ} \cap C_{1}\right|_{k}} \geq \frac{|P \cap C|_{k+1}\left|P^{\circ} \cap C\right|_{k+1}}{\left|P \cap C_{1}\right|_{k}\left|P^{\circ} \cap C_{1}\right|_{k}} .
$$

The main theorem of this section follows by repeated applications of the previous result:

Proof of Theorem 10: First we divide $K$ and $K^{\circ}$ into the cones generated by the facets of $P$. Let $F^{(n-1)}$ be one of these facets and let $C^{(n)}$ be the $n$-dimensional cone generated by $F^{(n-1)}$. Let $N$ be the number of facets of $P$. There exist $g_{1}, \ldots, g_{N} \in \mathcal{O}(P)$ such that the facets of $P$ are $g_{1} F^{(n-1)}, \ldots, g_{N} F^{(n-1)}$. One has thus $\mathbb{R}^{n}=\bigcup_{i=1}^{N} g_{i} C^{(n)}$ and since $K=g_{i} K$

$$
K=\bigcup_{i=1}^{N} K \cap g_{i} C^{(n)}=\bigcup_{i=1}^{N} g_{i}\left(K \cap C^{(n)}\right) .
$$

Since the union is essentially disjoint, we have $|K|=N\left|K \cap C^{(n)}\right|$. Recall that $K$ and $K^{\circ}$ have the same isometry group. Therefore we may apply the same for $K^{\circ}$, thus $\left|K^{\circ}\right|=N\left|K^{\circ} \cap C^{(n)}\right|$. Finally we get

$$
|K|_{n}\left|K^{\circ}\right|_{n}=N^{2}\left|K \cap C^{(n)}\right|_{n}\left|K^{\circ} \cap C^{(n)}\right|_{n}
$$

Note that in particular $|P|_{n}\left|P^{\circ}\right|_{n}=\left.N^{2}\left|P \cap C^{(n)}{ }_{n}\right| P^{\circ} \cap C^{(n)}\right|_{n}$. Let $\left(F^{(0)}, \ldots, F^{(n-1)}\right)$ be a maximal flag of $P$ and consider for $1 \leq j \leq n$ the $j$-dimensional cone $C^{(j)}=\operatorname{pos}\left(F^{(j-1)}\right)$. Denote by $\left(v_{i}^{(j)}\right)$ the collection of unit vectors which are orthogonal to the facets of $C^{(j)}$ and pointing inwards this cone. Since $C^{(k-1)}$ is one of the facets of $C^{(k)}$, Lemma 12 yields for $2 \leq k \leq n$

$$
\frac{\left|K \cap C^{(k)}\right|_{k}\left|K^{\circ} \cap C^{(k)}\right|_{k}}{\left|K \cap C^{(k-1)}\right|_{k-1}\left|K^{\circ} \cap C^{(k-1)}\right|_{k-1}} \geq \frac{\left|P \cap C^{(k)}\right|_{k}\left|P^{\circ} \cap C^{(k)}\right|_{k}}{\left|P \cap C^{(k-1)}\right|_{k-1}\left|P^{\circ} \cap C^{(k-1)}\right|_{k-1}}=\frac{\left|\sum_{i} v_{i}^{(k)}\right|^{2}}{k^{2}},
$$

and for $k=1$ simply $\left|K \cap C^{(1)}\right|_{1}\left|K^{\circ} \cap C^{(1)}\right|_{1}=\left|P \cap C^{(1)}\right|{ }_{1}\left|P^{\circ} \cap C^{(1)}\right|_{1}=1$. Taking the product of these inequalities one gets that

$$
|K|_{n}\left|K^{\circ}\right|_{n} \geq|P|_{n}\left|P^{\circ}\right|_{n}=N^{2} \prod_{k=2}^{n} \frac{\left|\sum_{i} v_{i}^{(k)}\right|^{2}}{k^{2}}=\left(\frac{N}{n !} \prod_{k=2}^{n}\left|\sum_{i} v_{i}^{(k)}\right|\right)^{2} .
$$

The barycenters of $P^{\circ}$ and $K^{\circ}$ are at this origin, because of their invariance by $\mathcal{O}(P)$. Hence $\mathcal{P}(K)=$ $|K|_{n}\left|K^{\circ}\right|_{n} \geq|P|_{n}\left|P^{\circ}\right|_{n}=\mathcal{P}(P)$. This proves the inequality.

If $\mathcal{P}(K)=\mathcal{P}(P)$ then there is equality in each of the preceding inequalities hence in particular in the first one, so from the equality case in Lemma $12, K \cap C^{(n)}$ is either a dilate of $P \cap C^{(n)}$ or of $P^{\circ} \cap C^{(n)}$. Since $K=\bigcup_{i=1}^{N} g_{i}\left(K \cap C^{(n)}\right)$, it follows that $K$ is either a dilate of $P$ or of $P^{\circ}$.

\section{$5 \quad$ Products of regular polytopes}

Given isometries $g_{\ell} \in \mathcal{O}\left(\mathbb{R}^{n_{\ell}}\right), \ell=1, \ldots, k$, we consider the isometry $g_{1} \times \cdots \times g_{k}$ defined on $\mathbb{R}^{n_{1}} \times \cdots \times \mathbb{R}^{n_{k}}$ by

$$
\left(g_{1} \times \cdots \times g_{k}\right)\left(x_{1}, \ldots, x_{k}\right)=\left(g_{1}\left(x_{1}\right), \ldots, g_{k}\left(x_{k}\right)\right) .
$$

For subgroups $G_{\ell} \subset \mathcal{O}\left(\mathbb{R}^{n_{\ell}}\right), \ell=1, \ldots, k$, we define their direct product as a subset of $\mathcal{O}\left(\mathbb{R}^{n}\right)$, where $n=\sum_{\ell} n_{\ell}:$

$$
G_{1} \times \cdots \times G_{k}=\left\{g_{1} \times \cdots \times g_{k} ; \forall i, g_{i} \in G_{i}\right\} .
$$

Our improved sharp lower bound on the volume products under symmetry assumptions is as follows: 
Theorem 13. Let $k \geq 1$ be an integer. For $1 \leq \ell \leq k$, let $P_{\ell}$ be a regular polytope in $\mathbb{R}^{n_{\ell}}$ with barycenter at the origin. Let $n=\sum_{\ell} n_{\ell}$ and $K \subset \mathbb{R}^{n}=\mathbb{R}^{n_{1}} \times \cdots \times \mathbb{R}^{n_{k}}$ be a convex body invariant by $\mathcal{O}\left(P_{1}\right) \times \cdots \times \mathcal{O}\left(P_{k}\right)$. Then

$$
\mathcal{P}(K) \geq \mathcal{P}\left(P_{1} \times \cdots \times P_{k}\right)=\frac{1}{n !} \prod_{\ell=1}^{k} n_{\ell} ! \mathcal{P}\left(P_{\ell}\right) .
$$

Taking $k=n$ and $P_{1}=\cdots=P_{n}=[-1,1]$, we recover the result of Saint Raymond [23] for unconditional sets in $\mathbb{R}^{n}$. The proof of this result will require most of the material of the previous sections. We start with a classical fact on the volume products of product-sets, which establishes the equality in the statement of the theorem.

Lemma 14. For $\ell=1, \ldots, k$, let $K_{\ell} \subset \mathbb{R}^{n_{\ell}}$ be a convex body containing the origin in its interior. Let $n=\sum_{\ell} n_{\ell}$. Then

$$
n !\left|K_{1} \times \cdots \times K_{k}\right|\left|\left(K_{1} \times \cdots \times K_{k}\right)^{\circ}\right|=\prod_{\ell=1}^{k}\left(n_{\ell} !\left|K_{\ell}\right|\left|K_{\ell}^{\circ}\right|\right) .
$$

Proof. Recall that the gauge function for $K_{\ell}^{\circ}$ is defined by $\|x\|_{K_{\ell}^{\circ}}=\sup _{y \in K_{\ell}}\langle x, y\rangle$. With this notation

$$
\begin{aligned}
\left(K_{1} \times \cdots \times K_{k}\right)^{\circ} & =\left\{\left(x_{1}, \ldots, x_{k}\right) \in \mathbb{R}^{n} ; \forall y_{1} \in K_{1}, \ldots, \forall y_{k} \in K_{k}, \sum_{\ell}\left\langle x_{\ell}, y_{\ell}\right\rangle \leq 1\right\} \\
& =\left\{\left(x_{1}, \ldots, x_{k}\right) \in \mathbb{R}^{n} ; \sum_{\ell}\left\|x_{\ell}\right\|_{K_{\ell}^{\circ}} \leq 1\right\} .
\end{aligned}
$$

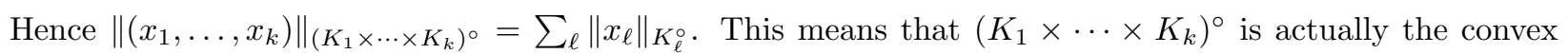

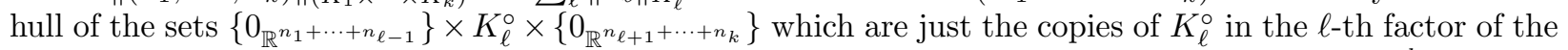
product space. We conclude using the following consequence of level-sets integration: if $K \subset \mathbb{R}^{d}$ is convex and contains the origin in its interior, then $d !|K|=\int_{\mathbb{R}^{d}} e^{-\|x\|_{K}} d x$. This gives

$$
\begin{aligned}
n !\left|\left(K_{1} \times \cdots \times K_{k}\right)^{\circ}\right| & =\int_{\mathbb{R}^{n_{1}} \times \cdots \times \mathbb{R}^{n_{k}}} e^{-\sum_{\ell=1}^{k}\left\|x_{\ell}\right\|_{\left(K_{\ell}\right)^{\circ}}} d x_{1} \ldots d x_{k} \\
& =\prod_{\ell=1}^{k} \int_{\mathbb{R}_{n_{\ell}}} e^{-\left\|x_{\ell}\right\|_{\left(K_{\ell}\right)^{\circ}}} d x_{\ell}=\prod_{\ell=1}^{k} n_{\ell} !\left|K_{\ell}^{\circ}\right|
\end{aligned}
$$

and we conclude by using that $\left|K_{1} \times \cdots \times K_{k}\right|=\prod_{\ell=1}^{k}\left|K_{i}\right|$.

Proof of Theorem 13: We first divide $\mathbb{R}^{n}$ into cones. For each $\ell \in\{1, \ldots, k\}$, let $N_{\ell}$ be the number of facets of $P_{\ell}$. Let also $\left(F_{\ell}^{0}, \ldots, F_{\ell}^{n_{\ell}-1}\right)$ be a maximal flag of $P_{\ell}$. We set $C_{\ell}^{0}=\left\{0_{\mathbb{R}^{n_{\ell}}}\right\}$ and for $i \in\left\{1, \ldots, n_{\ell}\right\}$, $C_{\ell}^{i+1}=\operatorname{pos}\left(F_{\ell}^{i}\right)$. With this definition $\left(C_{\ell}^{0}, C_{\ell}^{1}, \ldots, C_{\ell}^{n_{\ell}}\right)$ can be viewed as a maximal flag of the cone $C_{\ell}^{n_{\ell}}$. Note that $C_{\ell}^{i}$ is of dimension $i$.

As we have already seen, $\mathbb{R}^{n_{\ell}}$ can be essentially paved by the cones generated by the facets of $P_{\ell}$, which are all isometric. In other words, there exists isometries $g_{\ell, 1}, \ldots, g_{\ell, N_{\ell}} \in \mathcal{O}\left(P_{\ell}\right)$ such that

$$
\mathbb{R}^{n_{\ell}}=\cup_{j=1}^{N_{\ell}} g_{\ell, j}\left(C_{\ell}^{n_{\ell}}\right)
$$

where the overlaps have no volume. Taking cartesian products, we obtain that

$$
\begin{aligned}
\mathbb{R}^{n} & =\left(\bigcup_{j_{1}=1}^{N_{1}} g_{1, j_{1}}\left(C_{1}^{n_{1}}\right)\right) \times \cdots \times\left(\bigcup_{j_{k}=1}^{N_{k}} g_{k, j_{k}}\left(C_{k}^{n_{k}}\right)\right) \\
& =\bigcup_{1 \leq j_{1} \leq N_{1}, \ldots, 1 \leq j_{k} \leq N_{k}}\left(g_{1, j_{1}} \times \cdots \times g_{k, j_{k}}\right)\left(C_{1}^{n_{1}} \times \cdots \times C_{k}^{n_{k}}\right) .
\end{aligned}
$$


Since $K$ and $K^{\circ}$ are invariant by each $g_{1, j_{1}} \times \cdots \times g_{k, j_{k}} \in \mathcal{O}\left(P_{1}\right) \times \cdots \times \mathcal{O}\left(P_{k}\right)$, taking volumes gives

$$
|K|\left|K^{\circ}\right|=\left(N_{1} \cdots N_{k}\right)^{2}\left|K \cap\left(C_{1}^{n_{1}} \times \cdots \times C_{k}^{n_{k}}\right)\right|\left|K^{\circ} \cap\left(C_{1}^{n_{1}} \times \cdots \times C_{k}^{n_{k}}\right)\right| .
$$

Next, we follow Meyer's approach to estimate the volume product "in this cone" in terms of the volume product in its facets and so on. This will lead us to consider all the faces of $C_{1}^{n_{1}} \times \cdots \times C_{k}^{n_{k}}$ of any dimension. It is easy to see that they are all of the form $C(1) \times \cdots \times C(k)$ where $C(\ell)$ is a face of $C_{\ell}^{n_{\ell}}$ (meaning either $\{0\}$ or the positive hull of a face of $P_{\ell}$ ). In particular, since the group of isometries of $P_{\ell}$ is transitive on the faces of $P_{\ell}$ of a given dimension, all the cones that will appear in our proof will be isometric, by an element of $\mathcal{O}\left(P_{1}\right) \times \cdots \times \mathcal{O}\left(P_{k}\right)$, to a cone of the form $C_{1}^{d_{1}} \times \cdots \times C_{k}^{d_{k}}$, with $0 \leq d_{\ell} \leq n_{\ell}$. Note that $d_{1}+\cdots+d_{k}$ is the dimension of this cone. Now there may be many different cones of a given dimension $d$, as many as the choices of $\left(d_{\ell}\right)$ such that $d_{1}+\cdots+d_{k}=d$.

Lemma 15. Let $K \subset \mathbb{R}^{n}$ be a convex body, which is assumed to be $\mathcal{O}\left(P_{1}\right) \times \cdots \times \mathcal{O}\left(P_{k}\right)$-invariant. For each $\ell \in\{1, \ldots, k\}$, let $D_{\ell}$ be $\left\{0_{\mathbb{R}^{n}}\right\}$ or the positive hull of a face of $P_{\ell}$. Let $D=D_{1} \times \cdots \times D_{k}$ and assume that it is of dimension $d \geq 1$. If $\operatorname{dim}\left(D_{\ell}\right) \geq 1$, let $D_{\ell, 1}, \ldots, D_{\ell, j_{\ell}}$ be its facets and let $v_{\ell, 1}, \ldots, v_{\ell, j_{\ell}}$ be the unit inner normals to these facets. We also set $w\left(D_{\ell}\right):=v_{\ell, 1}+\ldots+v_{\ell, j_{\ell}}$ and define the $(d-1)$-dimensional cone

$$
\tilde{D}_{\ell}:=D_{1} \times \cdots \times D_{\ell-1} \times D_{\ell, 1} \times D_{\ell+1} \times \cdots \times D_{k} .
$$

With these notations the following inequality holds

$$
d^{2}|K \cap D|\left|K^{\circ} \cap D\right| \geq \sum_{\ell=1, \ldots, k, \operatorname{dim}\left(D_{\ell}\right)>0}\left|w\left(D_{\ell}\right)\right|^{2}\left|K \cap \tilde{D}_{\ell}\right|\left|K^{\circ} \cap \tilde{D}_{\ell}\right| .
$$

We could set $w\left(D_{\ell}\right)=0$ when $\operatorname{dim}\left(D_{\ell}\right)=0$ and this would allow to remove the restriction in the sum.

Proof. The facets of the cone $D=D_{1} \times \cdots \times D_{k}$ are the cones

$$
D_{1} \times \cdots \times D_{\ell-1} \times D_{\ell, j} \times D_{\ell+1} \times \cdots \times D_{k}
$$

for all the choices of $\ell$ such that $\operatorname{dim}\left(D_{\ell}\right) \geq 1$ and of $j \in\left\{1, \ldots, j_{\ell}\right\}$. The unit normal to this facet of $D$ pointing inside $D$ is simply $v_{\ell, j}$ viewed as a vector in $\mathbb{R}^{n}$. This means that from now on it is identified with the vector

$$
\left(0_{\mathbb{R}^{n_{1}}}, \ldots, 0_{\mathbb{R}^{n_{\ell-1}}}, v_{\ell, j}, 0_{\mathbb{R}^{n_{\ell+1}}}, \ldots, 0_{\mathbb{R}^{n_{k}}}\right) .
$$

In particular for different values of $\ell$ the normal vectors are orthogonal. The usual volume estimate yields for all $x \in K \cap D$ that

$$
\begin{aligned}
|K \cap D| & \geq \sum_{\ell, \operatorname{dim}\left(D_{\ell}\right)>0} \sum_{j=1}^{j_{\ell}}\left|\operatorname{conv}\left(x, K \cap\left(D_{1} \times \cdots \times D_{\ell-1} \times D_{\ell, j} \times D_{\ell+1} \times \cdots \times D_{k}\right)\right)\right| \\
& =\sum_{\ell, \operatorname{dim}\left(D_{\ell}\right)>0} \sum_{j=1}^{j_{\ell}} \frac{\left\langle x, v_{\ell, j}\right\rangle}{d}\left|K \cap\left(D_{1} \times \cdots \times D_{\ell-1} \times D_{\ell, j} \times D_{\ell+1} \times \cdots \times D_{k}\right)\right| .
\end{aligned}
$$

Now for all $(\ell, j)$ in the above sum, there exists $g \in \mathcal{O}\left(P_{\ell}\right)$ such that $g\left(D_{\ell, j}\right)=D_{\ell, 1}$ as we explained in the section about regular polytopes. Hence

$$
i_{\ell}[g]\left(D_{1} \times \cdots \times D_{\ell-1} \times D_{\ell, j} \times D_{\ell+1} \times \cdots \times D_{k}\right)=D_{1} \times \cdots \times D_{\ell-1} \times D_{\ell, 1} \times D_{\ell+1} \times \cdots \times D_{k}=\tilde{D}_{\ell},
$$

and since $K$ is invariant by $i_{\ell}[g]$ we get that $\left|K \cap\left(D_{1} \times \cdots \times D_{\ell-1} \times D_{\ell, j} \times D_{\ell+1} \times \cdots \times D_{k}\right)\right|=\left|K \cap \tilde{D}_{\ell}\right|$. Hence

$$
|K \cap D| \geq \sum_{\ell, \operatorname{dim}\left(D_{\ell}\right)>0} \sum_{j=1}^{j_{\ell}} \frac{\left\langle x, v_{\ell, j}\right\rangle}{d}\left|K \cap \tilde{D}_{\ell}\right|=\sum_{\ell, \operatorname{dim}\left(D_{\ell}\right)>0} \frac{\left|K \cap \tilde{D}_{\ell}\right|}{d}\left\langle x, w\left(D_{\ell}\right)\right\rangle .
$$


So the vector

$$
y:=\sum_{\ell, \operatorname{dim}\left(D_{\ell}\right)>0} \frac{\left|K \cap \tilde{D}_{\ell}\right|}{d|K \cap D|} w\left(D_{\ell}\right)
$$

satisfies that $\sup _{x \in K \cap D}\langle x, y\rangle \leq 1$. We have seen in the previous section that $w\left(D_{\ell}\right) \in D_{\ell}$ (again we identify

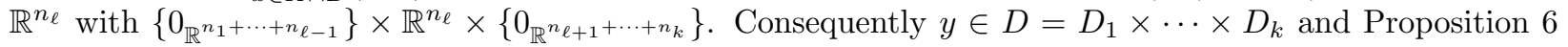
implies that $y \in K^{\circ}$. Since $y \in K^{\circ} \cap D$, we may reproduce the above volumic argument and get

$$
\begin{aligned}
\left|K^{\circ} \cap D\right| & \geq \sum_{m, \operatorname{dim}\left(D_{m}\right)>0} \frac{\left|K^{\circ} \cap \tilde{D}_{m}\right|}{d}\left\langle y, w\left(D_{m}\right)\right\rangle \\
& =\sum_{m, \operatorname{dim}\left(D_{m}\right)>0} \sum_{\ell, \operatorname{dim}\left(D_{\ell}\right)>0} \frac{\left|K^{\circ} \cap \tilde{D}_{m}\right|\left|K \cap \tilde{D}_{\ell}\right|}{d^{2}|K \cap D|}\left\langle w\left(D_{m}\right), w\left(D_{\ell}\right)\right\rangle
\end{aligned}
$$

Eventually, if $\ell \neq m, w\left(D_{\ell}\right)$ and $w\left(D_{m}\right)$ are orthogonal. The proof is therefore complete.

Continuation of the proof of Theorem 13. We start with recalling Equation (6)

$$
|K|\left|K^{\circ}\right|=\left(N_{1} \cdots N_{k}\right)^{2}\left|K \cap\left(C_{1}^{n_{1}} \times \cdots \times C_{k}^{n_{k}}\right)\right|\left|K^{\circ} \cap\left(C_{1}^{n_{1}} \times \cdots \times C_{k}^{n_{k}}\right)\right|,
$$

where $C_{\ell}^{0}=\left\{0_{\mathbb{R}_{\ell} n_{\ell}}\right\}$ and for $j \geq 1, C_{\ell}^{j}=\operatorname{pos}\left(F_{\ell}^{j-1}\right)$ and $\left(F_{\ell}^{0}, \ldots, F_{\ell}^{n_{\ell}-1}\right)$ is a maximal facial flag of $P_{\ell}$. Since the origin is the only point that is fixed by the whole group $\mathcal{O}\left(P_{1}\right) \times \cdots \times \mathcal{O}\left(P_{k}\right)$, which leaves $K$ and $K^{\circ}$ globally invariant, it follows that 0 is the barycenter of $K^{\circ}$. Hence $\mathcal{P}(K)=|K|\left|K^{\circ}\right|$ and our task is to show that $|K|\left|K^{\circ}\right| \geq\left|P_{1} \times \cdots \times P_{k}\right|\left|\left(P_{1} \times \cdots \times P_{k}\right)^{\circ}\right|$. In view of Lemma 14 and Formula (5), this amounts to

$$
n !|K|\left|K^{\circ}\right| \geq \prod_{\ell=1}^{k} \frac{N_{\ell}^{2}}{n_{\ell} !} \prod_{j_{\ell}=2}^{n_{\ell}}\left|w\left(C_{\ell}^{j_{\ell}}\right)\right|^{2}
$$

Note that $\left|w\left(C_{\ell}^{1}\right)\right|=1$ since a half-line has only one boundary point and one inner normal. The latter is equivalent to

$$
n !\left|K \cap\left(C_{1}^{n_{1}} \times \cdots \times C_{k}^{n_{k}}\right)\right|\left|K^{\circ} \cap\left(C_{1}^{n_{1}} \times \cdots \times C_{k}^{n_{k}}\right)\right| \geq \prod_{\ell=1}^{k} \prod_{j_{\ell}=1}^{n_{\ell}} \frac{\left|w\left(C_{\ell}^{j_{\ell}}\right)\right|^{2}}{j_{\ell}} .
$$

We prove this inequality by repeated applications of Lemma 15. Let us introduce more compact notations. Given a cone $D$ of dimension $d$ we set $[D]:=(d !)^{2}|K \cap D|\left|K^{\circ} \cap D\right|$ and $\lambda(D):=|w(D)|^{2} / d$. If $D$ is reduced to the origin we take by convention $\lambda(D)=1$. Our goal is to show by induction on $d$, where $1 \leq d=d_{1}+\cdots+d_{k}$, with $d_{i} \leq n_{i}$, that

$$
\left[C_{1}^{d_{1}} \times \cdots \times C_{k}^{d_{k}}\right] \geq\left(d_{1}+\cdots+d_{k}\right) ! \prod_{\ell=1}^{k} \prod_{j_{\ell}=1}^{d_{\ell}} \lambda\left(C_{\ell}^{j_{\ell}}\right) .
$$

The starting point is that if $d=d_{1}+\cdots+d_{k}=1$, then $C_{1}^{d_{1}} \times \cdots \times C_{k}^{d_{k}}$ is a half-line with the "norming" property, hence $\left[C_{1}^{d_{1}} \times \cdots \times C_{k}^{d_{k}}\right]=1$ by the usual argument. The induction argument is provided by Lemma 15 which implies that

$$
\left[C_{1}^{d_{1}} \times \cdots \times C_{k}^{d_{k}}\right] \geq \sum_{\ell=1}^{k} d_{\ell} \lambda\left(C_{\ell}^{d_{\ell}}\right)\left[C_{1}^{d_{1}} \times \cdots \times C_{\ell-1}^{d_{\ell-1}} \times C_{\ell}^{d_{\ell}-1} \times C_{\ell+1}^{d_{\ell+1}} \times \cdots \times C_{k}^{d_{k}}\right]
$$


provided $d=d_{1}+\cdots+d_{k} \geq 1$. If we know the required property for product cones of dimension $d-1 \geq 1$, then we know the following lower bound for each term of the right-hand side of the above inequality:

$$
\begin{aligned}
& {\left[C_{1}^{d_{1}} \times \cdots \times C_{\ell-1}^{d_{\ell-1}} \times C_{\ell}^{d_{\ell}-1} \times C_{\ell+1}^{d_{\ell+1}} \times \cdots \times C_{k}^{d_{k}}\right] } \\
\geq & \left(d_{1}+\cdots+d_{k}-1\right) ! \prod_{i \neq \ell}\left(\prod_{j_{i}=1}^{d_{i}} \lambda\left(C_{i}^{j_{i}}\right)\right) \times \prod_{j_{\ell}=1}^{d_{\ell}-1} \lambda\left(C_{\ell}^{j_{\ell}}\right) \\
= & \left(d_{1}+\cdots+d_{k}-1\right) ! \frac{\prod_{i=1}^{k}\left(\prod_{j_{i}=1}^{d_{i}} \lambda\left(C_{i}^{j_{i}}\right)\right)}{\lambda\left(C_{\ell}^{d_{\ell}}\right)} .
\end{aligned}
$$

Plugging these inequalities in (8) gives

$$
\left[C_{1}^{d_{1}} \times \cdots \times C_{k}^{d_{k}}\right] \geq \sum_{\ell=1}^{k} d_{\ell}\left(d_{1}+\cdots+d_{k}-1\right) ! \prod_{i=1}^{k} \prod_{j_{i}=1}^{d_{i}} \lambda\left(C_{i}^{j_{i}}\right)=\left(d_{1}+\cdots+d_{\ell}\right) ! \prod_{i=1}^{k} \prod_{j_{i}=1}^{d_{i}} \lambda\left(C_{i}^{j_{i}}\right) .
$$

After $n-1$ iteration, one reaches (7). Alternatively we could have checked that the inequality provided by Lemma 15 is always an equality when $K=P_{1} \times \cdots \times P_{k}$ and check that after $n-1$ iterations it gives a lower bound on the volume product of $K$ by a quantity that does not depend on $K$ anymore.

Remark 5. The inequality provided by Theorem 13 is an equality when $K=P_{1} \times \cdots \times P_{k}$ but there are many more equality cases. We illustrate this in the case when $k=2$. It is convenient to introduce for $K \subset \mathbb{R}^{n_{1}}$ and $M \subset \mathbb{R}^{n_{2}}$, the set $K \diamond M:=\operatorname{conv}\left(K \times\left\{0_{\mathbb{R}^{n_{2}}}\right\},\left\{0_{\mathbb{R}^{n_{1}}}\right\} \times M\right)$. Note that when $K$ and $M$ contain the origin, $(K \times M)^{\circ}=K^{\circ} \diamond M^{\circ}$. Using this remark and noting that $\mathcal{P}\left(P_{i}\right)=\mathcal{P}\left(P_{i}^{\circ}\right)$ one readily checks that the sets $P_{1} \times P_{2}, P_{1}^{\circ} \times P_{2}, P_{1} \times P_{2}^{\circ}, P_{1}^{\circ} \times P_{2}^{\circ}, P_{1} \diamond P_{2}, P_{1}^{\circ} \diamond P_{2}, P_{1} \diamond P_{2}^{\circ}$ and $P_{1}^{\circ} \diamond P_{2}^{\circ}$ are all $\mathcal{O}\left(P_{1}\right) \times \mathcal{O}\left(P_{2}\right)$-invariant and have minimal volume product in this invariance class.

For $k \geq 3$, one has to deal moreover with parentheses since for example $\left(P_{1} \times P_{2}\right) \diamond P_{3} \neq P_{1} \times\left(P_{2} \diamond P_{3}\right)$, as can be seen by comparing the vertices of each set. Hence one can produce minimizers by arbitrary parentheses combinations of cartesian products $\times$ and cartesian convex hulls $\diamond$ of the sets $P_{i}$ and their polars. In the case of unconditional bodies, $\left(P_{\ell}=[-1,1]\right.$ for all $\left.\ell\right)$, Meyer [14] and independently with another method Reisner [22] were able to show that all unconditional minimal volume product bodies are of this form (they are known as Hanner polytopes). It should be possible, but tedious indeed, to extend Meyer's argument to general $P_{\ell}$ 's in order to characterize equality cases in Theorem 13.

\section{$6 \quad$ General symmetries}

The following result confirms Mahler's conjecture for convex bodies with many hyperplane symmetries.

Theorem 16. Let $K \subset \mathbb{R}^{n}$ be a convex body. Assume that there exists $m$ (non necessarily orthogonal) symmetries $S_{1}, \ldots, S_{m}$ with respect to affine hyperplanes $H_{1}, \ldots, H_{m}$ such that for all $i=1, \ldots, m, S_{i}(K)=$ $K$ and $\operatorname{card}\left(\bigcap_{i=1}^{m} H_{i}\right)=1$. Then

$$
\mathcal{P}(K) \geq \mathcal{P}\left(\Delta_{n}\right)
$$

with equality if and only if $K$ is a simplex.

This theorem will be deduced from our previous results using the following structural statement, which relies on Coxeter's classification of finite reflection groups [3] (see also [4] and [9]).

Proposition 17. Let $K \subset \mathbb{R}^{n}$ be a compact set. Let $\mathcal{R}(K)$ be the subgroup of $\mathcal{O}\left(\mathbb{R}^{n}\right)$ generated by the symmetries with respect to vector hyperplanes which leave $K$ globally invariant. Assume that $\operatorname{Fix}(\mathcal{R}(K))=$ $\{0\}$. Then there exists an orthogonal decomposition $\mathbb{R}^{n}=E_{1} \oplus \cdots \oplus E_{k}$ and convex sets $P_{\ell} \subset E_{\ell}, \ell=$ $1, \ldots, k$ such that $P_{\ell}$ is a regular polygon if $\operatorname{dim}\left(E_{\ell}\right)=2$ and a regular simplex of dimension $\operatorname{dim}\left(E_{\ell}\right)$ otherwise, and

$$
\mathcal{O}\left(P_{1}\right) \times \cdots \times \mathcal{O}\left(P_{k}\right) \subset \mathcal{R}(K) .
$$


Remark 6. In the previous statement, $E_{\ell}$ may be of dimension 1 and therefore identified to $\mathbb{R}$. In this case $P_{\ell}=\Delta_{1}=[-1,1]$ and $\mathcal{O}([-1,1])=\left\{\operatorname{Id}_{\mathbb{R}},-\operatorname{Id}_{\mathbb{R}}\right\}$. Note that the direct product of $n$ copies of $\mathcal{O}([-1,1])$ corresponds to the reflection group generated by $n$ hyperplane symmetries with respect to the coordinate hyperplanes in an orthonormal basis. By definition $K \subset \mathbb{R}^{n}$ is unconditional if and only if, in an appropriate orthonormal basis, it is invariant by $\left(x_{1}, \ldots, x_{n}\right) \mapsto\left( \pm x_{1}, \ldots, \pm x_{n}\right)$. This is clearly equivalent to being invariant by the direct product $\mathcal{O}([-1,1]) \times \cdots \times \mathcal{O}([-1,1])$ (in the orthogonal decomposition induced by the basis).

Proof. The first step of the proof is very classical. It consists in writing $\mathcal{R}(K)$ as a direct product of irreducible reflection groups. Recall that a reflection group acting on $\mathbb{R}^{n}$ is reducible if there exists a vector subspace $E \subset \mathbb{R}^{n}$ with $\{0\} \neq E \neq \mathbb{R}^{n}$ such that $E$ is globally invariant for all the elements of $\mathcal{R}(K)$. Consequently $E^{\perp}$ is also globally invariant. If a reflection $S_{H}$ with respect to a hyperplane $H=\{h\}^{\perp}$ leaves $E$ globally invariant then either $h \in E$ or $h \in E^{\perp}$ (indeed for all $v \in E, 2\langle v, h\rangle h=v-S_{H} v \in E$ ). Hence, in the decomposition $\mathbb{R}^{n}=E \bigoplus E^{\perp}$, either $S_{H}=S_{h^{\perp E}} \times \operatorname{Id}_{E \perp}$ or $S_{H}=\operatorname{Id}_{E} \times S_{h^{\perp E} \perp}$ where $A^{\perp E}$ stands for the orthogonal of a set $A$ in $E$. It is then easy to see that the $\mathrm{R}(K)$ being generated by reflections becomes a direct product of a subgroup of $\mathcal{O}(E)$ generated by reflections and a subgroup of $\mathcal{O}\left(E^{\perp}\right)$ generated by reflections. Repeating this procedure whenever possible leads to an orthogonal decomposition $\mathbb{R}^{n}=\bigoplus_{i=1}^{m} E_{i}$ in which $\mathcal{R}(K)=\mathcal{R}_{1} \times \cdots \times \mathcal{R}_{m}$ where for all $i, \mathcal{R}_{i}$ is an irreducible reflection group on $E_{i} \sim \mathbb{R}^{\operatorname{dim}\left(E_{i}\right)}$. In order to prove the claim it is sufficient to show that every $\mathcal{R}_{i}$ contains a subgroup of the form $\mathcal{O}\left(P_{1}\right) \times \cdots \times \mathcal{O}\left(P_{k}\right)$ where each $P_{k}$ is either a regular simplex or a regular polygon. If $\mathcal{R}_{i}$ is infinite, one can show that $\mathcal{R}_{i}=\mathcal{O}\left(E_{i}\right)$ (this is postponed to the next lemma) hence it contains the symmetries with respect to the hyperplanes corresponding to an orthonormal basis, i.e. $\mathcal{O}([-1,1]) \times \cdots \times \mathcal{O}([-1,1]) \subset \mathcal{R}_{i}$ and we are done with this factor. If $\mathcal{R}_{i}$ is finite we use the complete description of finite irreducible reflection groups, due to Coxeter [3]. For each such group we show that it contains a direct product of isometry groups of regular polygons or simplices. We proceed as follows: we consider first the groups which may be viewed as isometry groups of regular polytopes. The remaining cases are isometry groups of semi-regular polytopes. In each category, we consider first the cases which are specific to small dimensions and study next the families of groups existing in any dimension. The finite irreducible reflection groups corresponding to regular polytopes are:

- The diedral groups $I_{2}(m), m \geq 2$ may be viewed as the isometry groups of the regular polytopes $\mathcal{C}_{m}$. They are of the form expected in the Proposition.

- The group $\mathrm{H}_{3}$ is the group of isometries of the dodecahedron and of its polar the icosahedron. Let $\varphi=(1+\sqrt{5}) / 2$. Then an icosahedron can be built as the convex hull of the set of points obtained from $(0,1, \varphi) \in \mathbb{R}^{3}$ by permutations of the coordinates and arbitrary changes of signs. Therefore the icosahedron is unconditional for the canonical basis. Hence its isometry group contains $\mathcal{O}([-1,1]) \times$ $\mathcal{O}([-1,1]) \times \mathcal{O}([-1,1])$ and we are done with this case.

- The group $F_{4}$ is the isometry group of the $\operatorname{set} \operatorname{conv}\left(B_{\infty}^{4}, 2 B_{1}^{4}\right) \subset \mathbb{R}^{4}$, usually called the 24-cell. This set is clearly unconditional for the canonical basis. Hence $F_{4}$ contains a subgroup of the expected shape.

- The group $H_{4}$ is the isometry group of the 120 -cell and its polar the 600 -cell. These two subsets of $\mathbb{R}^{4}$ turn out to be unconditional as well. Indeed one can build a 600 -cell by taking the convex hull of the 24-cell $\operatorname{conv}\left(B_{\infty}^{4}, 2 B_{1}^{4}\right)$ and all the points obtained from $(1, \varphi, 1 / \varphi, 0)$ by arbitrary changes of signs and even permutations. Hence $H_{4}$ contains the direct product of four copies of $\mathcal{O}([-1,1])$.

- The group $A_{n}$ way be viewed as the isometry group of a regular simplex in $\mathbb{R}^{n}$. It is of the form predicted in the proposition.

- The group $B_{n}$ also called $C_{n}$ corresponds to the isometry group of the $n$-dimensional cube $B_{\infty}^{n}=$ $[-1,1]^{n}$ and its dual $B_{1}^{n}$. The cube being unconditional $B_{n}$ contains a direct product of $n$ copies of $\mathcal{O}([-1,1])$.

The remaining cases only correspond to isometry groups of semi-regular polytopes. 
- We deal with the group $E_{6}$ in a different way: namely by considering its roots system. The roots are basically orthogonal vectors to the hyperplanes corresponding to the reflections in the group. In particular, a reflection group splits as a direct product if and only if its roots can be partitioned into two sets which are mutually orthogonal. Along the same ideas the existence of an orthogonal basis formed by roots ensures that the group contains a copy of $\mathcal{O}([-1,1]) \times \cdots \times \mathcal{O}([-1,1])$. The group $E_{6}$ is a reflection group acting on $\mathbb{R}^{6}$. Nevertheless it is convenient to use a representation of its roots in a subspace of dimension 6 in $\mathbb{R}^{9}$. Among the list of roots, let us just focus on the following ones:

$$
\begin{array}{ll}
v_{1,1}=(-1,1,0,0,0,0,0,0,0), & v_{1,2}=(0,1,-1,0,0,0,0,0,0) \\
v_{2,1}=(0,0,0,-1,1,0,0,0,0), & v_{2,2}=(0,0,0,0,1,-1,0,0,0) \\
v_{3,1}=(0,0,0,0,0,0,-1,1,0), & v_{2,2}=(0,0,0,0,0,0,0,1,-1) .
\end{array}
$$

Note that the three planes $\operatorname{span}\left(v_{i, 1}, v_{i, 2}\right), i \in\{1,2,3\}$ are mutually orthogonal. More over for each $i, \cos \left(\left(\widehat{v_{i, 1}, v_{i, 2}}\right)\right)=\frac{1}{2}$. So the angle between these vectors is $\pi / 3$ and so is the angle between their orthogonal hyperplanes. Hence the reflections corresponding to these two roots generate a copy of the $I_{2}(3)$ the group of isometries of the equilateral triangle. As a consequence $E_{6}$ contains $\mathcal{O}\left(\Delta_{2}\right) \times$ $\mathcal{O}\left(\Delta_{2}\right) \times \mathcal{O}\left(\Delta_{2}\right)$.

- The system of roots of the group $E_{7}$ acting on $\mathbb{R}^{7}$ is better presented in $\left\{x \in \mathbb{R}^{8} ; \sum_{i} x_{i}=0\right\}$. It consists of all the permutations of the vector $(1,-1,0,0,0,0,0,0)$ and all the permutations of $\frac{1}{2}(1,1,1,1,-1,-1,-1,-1)$. We just observe that among these vectors, the following seven vectors are orthogonal:

$$
\begin{aligned}
& (1,-1,0,0,0,0,0,0),(0,0,1,-1,0,0,0,0),(0,0,0,0,1,-1,0,0),(0,0,0,0,0,0,1,-1) \\
& \frac{1}{2}(1,1,1,1,-1,-1,-1,-1), \frac{1}{2}(1,1,-1,-1,1,1,-1,-1), \frac{1}{2}(1,1,-1,-1,-1,-1,1,1) .
\end{aligned}
$$

Hence $E_{7}$ contains a product of 7 copies of $\mathcal{O}([-1,1])$.

- The root system of the group $E_{8}$ contains in particular all the vectors obtained from $(1,1,0,0,0,0,0,0) \in$ $\mathbb{R}^{8}$ by changes of signs and permutations. Hence it contains 8 orthogonal vectors:

$$
e_{1}+e_{2}, e_{1}-e_{2}, e_{3}+e_{4}, e_{3}-e_{4}, e_{5}+e_{6}, e_{5}-e_{6}, e_{7}+e_{8}, e_{7}-e_{8}
$$

where $\left(e_{i}\right)_{i=1}^{8}$ is the canonical basis of $\mathbb{R}^{8}$. So $E_{8}$ contains the direct product of 8 copies of $\mathcal{O}([-1,1])$.

- The group $D_{n}$ exists in any dimension but provides a new object only for $n \geq 5$. It is the isometry group of the "demihypercube"

$$
D H^{n}:=\operatorname{conv}\left\{x \in\{-1,1\}^{n} ; \prod_{i=1}^{n} x_{i}=1\right\} .
$$

So the demihypercube is the convex hull of the vertices of the cube which have an even number of negative coordinates. If $n \geq 5$ it has two kinds of facets (some are regular simplices, some are lower dimensional demihypercubes). Its isometry group $D_{n}$ is of cardinality $2^{n-1} n$ !. It can be described as follows: for any permutation $\sigma$ of $\{1, \ldots, n\}$ and any $A \subset\{1, \ldots, n\}$ of even cardinality, the map

$$
\left(x_{i}\right)_{i=1}^{n} \mapsto\left((-1)^{\mathbb{1}_{A}(i)} x_{\sigma(i)}\right)
$$

belongs to $D_{n}$ and all isometries are of this type. It is not hard to see that the hyperplanes of symmetry of the demihypercube are the hyperplanes $\left\{e_{i}+e_{j}\right\}^{\perp}$ and $\left\{e_{i}-e_{j}\right\}^{\perp}$ for all choices $1 \leq i<j \leq n$. They correspond to the maps

$$
\begin{aligned}
\left(x_{1}, \ldots, x_{i}, \ldots, x_{j}, \ldots, x_{n}\right) & \mapsto\left(x_{1}, \ldots,-x_{j}, \ldots,-x_{i}, \ldots, x_{n}\right) \\
\left(x_{1}, \ldots, x_{i}, \ldots, x_{j}, \ldots, x_{n}\right) & \mapsto\left(x_{1}, \ldots, x_{j}, \ldots, x_{i}, \ldots, x_{n}\right) .
\end{aligned}
$$


If $n=2 k$ is even, $D H^{n}$ is unconditional with respect to the orthogonal basis formed by the $n$ vectors $e_{2 j-1}+e_{2 j}, e_{2 j-1}-e_{2 j}, j=1, \ldots, k$, and we are done with $D_{2 k}$.

Eventually, assume that $n \geq 5$ is odd and set $n=2 k+3$. Note that

$$
D H^{3}=\operatorname{conv}\{(1,1,1),(1,-1,-1),(-1,1,-1),(-1,-1,1)\}
$$

is a regular tetrahedron (isometric to $\Delta_{3}$ ). The reflections of hyperplanes orthogonal to the vectors $e_{1} \pm e_{2}, e_{2} \pm e_{3}, e_{3} \pm e_{1}$ generate a group which acts as $\mathcal{O}\left(\Delta_{3}\right)$ on the first factor of $\mathbb{R}^{3} \times \mathbb{R}^{2 k}=\mathbb{R}^{n}$ and as the identity on the second factor. Next we note that the vectors $e_{3+2 j-1}+e_{3+2 j}, e_{3+2 j-1}-e_{3+2 j}$ for $j=1, \ldots, k$ form an orthogonal basis of $\left\{0_{\mathbb{R}^{3}}\right\} \times \mathbb{R}^{2 k}$. Since the symmetries with respect to the orthogonal to these vectors are in $D_{n}$ we may conclude that

$$
\mathcal{O}\left(\Delta_{3}\right) \times \underbrace{\mathcal{O}([-1,1]) \times \cdots \times \mathcal{O}([-1,1])}_{2 k} \subset D_{n}
$$

All the cases have been treated, hence the claim is proved.

The case of irreducible infinite reflection subgroups of $\mathcal{R}(K)$ was not treated. Since $K$ is closed, the limit of a converging sequence of hyperplane symmetries in $\mathcal{R}(K)$ is still in $\mathcal{R}(K)$. We take advantage of this topological assumption in the next statement, which is certainly classical.

Lemma 18. Let $\mathcal{R}$ be an irreducible reflection group acting on $\mathbb{R}^{n}$. We assume that $\mathcal{R}$ is infinite and that the set of reflections in $\mathcal{R}$ is closed. Then $\mathcal{R}=\mathcal{O}\left(\mathbb{R}^{n}\right)$.

Proof. Assume $n \geq 2$. We define $A:=\left\{h \in S^{n-1} ; S_{h^{\perp}} \in \mathcal{R}\right\}$. Our goal is to show that $A=S^{n-1}$ which would imply that all reflections are in $\mathcal{R}$, hence $\mathcal{R}=\mathcal{O}\left(\mathbb{R}^{n}\right)$. Our topological hypothesis implies that $A$ is closed.

The set of fixed points $\operatorname{Fix}(\mathcal{R})$ is invariant under the action of $\mathcal{R}$, it is also included in a hyperplane since $A$ is not empty. By irreducibility and since the group is generated by its reflections, it follows that

$$
\{0\}=\operatorname{Fix}(\mathcal{R})=\bigcap_{h \in A} h^{\perp} .
$$

Hence the vectors $\{h ; h \in A\}$ span $\mathbb{R}^{n}$.

Next for all $R \in \mathcal{R}$ and $h \in A, S_{(R h)^{\perp}}=R^{-1} S_{h^{\perp}} R$ belongs to $\mathcal{R}$. Hence $R(A) \subset A$ and the restriction of $R$ to $A$ is a permutation. Since $A$ spans $\mathbb{R}^{n}$, it follows that the map $R \in \mathcal{R} \mapsto R_{\mid A}$ is injective into the set of permutations of $A$. As $\mathcal{R}$ is infinite, so is $A$.

Since $A$ is compact and infinite, there exists a sequence $\left(v_{n}\right)_{n}$ in $A$ converging to $v \in A$ and such that $v_{n} \neq v$ for all $n \geq 0$. Passing to a subsequence we can also assume that the sequence of unit vectors $\left(v_{n}-v\right) /\left|v_{n}-v\right|$ converges to a vector $w \in S^{n-1}$. Note that $w$ is orthogonal to $v$. Next we use the following observation: if $u_{1}, u_{2} \in A$ then $S_{u_{2}^{\frac{1}{2}}} \circ S_{u_{1}^{\perp}} \in \mathcal{R}$ is the rotation of angle $2 \alpha:=2\left(\widehat{u_{1}, u_{2}}\right)$ in the plane spanned by $u_{1}, u_{2}$ and the identity on the orthogonal. Hence the iterated images of $u_{1}$ by this rotation are in $A$. Consequently, any point from $S^{n-1} \cap \operatorname{span}\left(u_{1}, u_{2}\right)$ is at most at (geodesic) distance $\alpha$ from $A$. Let us apply this to $v$ and $v_{n}$ as $n$ tends to infinity. Since their angle tends to zero and since their span tends to $\operatorname{span}(v, w)$, the closedness of $A$ yields that $S^{n-1} \cap \operatorname{span}(v, w) \subset A$. Hence $A$ contains a one-dimensional sphere isometric to $S^{1}$. So we are done if $n=2$. If $n>2$ we show inductively that for all $k \in\{2, \ldots, n-1\}, A$ contains an isometric copy of $S^{k}$. The case $k=n-1$ is exactly our goal. Here is how we pass from $k \in\{1, \ldots, n-2\}$ to $k+1$ : Assume that there is a $k+1$-dimensional vector space $E \subset \mathbb{R}^{n}$ such that $\mathcal{S}:=E \cap S^{n-1} \subset A$. Since $A$ spans the whole space and cannot be split into two orthogonal parts, there exists $z \in S^{n-1}$ with $z \notin E \cup E^{\perp}$. Let $v \in S$ and choose $w \in S$ orthogonal to $v$ and such that $z$ is not orthogonal to $\operatorname{span}(v, w)$ (this is possible, otherwise $z$ would be orthogonal to $E$ ). Consider $v(\theta)=\cos (\theta) v+\sin (\theta) w$ and the function

$$
f(\theta)=\langle v(\theta), z\rangle=\sqrt{\langle v, z\rangle^{2}+\langle w, z\rangle^{2}} \cos (\theta-\varphi),
$$


where $\varphi=\varphi(v, w, z)$ is obviously defined. Since $z$ is not orthogonal to $\operatorname{span}(v, w),\langle v, z\rangle^{2}+\langle w, z\rangle^{2} \neq 0$. It is easily checked that $\{\theta \in \mathbb{R} ; f(\theta) \in \cos (\mathbb{Q} \pi)\}$ is countable. Consequently

$$
\Theta:=\{\theta \in \mathbb{R} ;(\widehat{v(\theta), z}) \notin \pi \mathbb{Q}\}
$$

is dense. In particular 0 is in its closure. As we already observed, the iterated images of $v(\theta)$ by the rotation of angle $\alpha:=2(\widehat{v(\theta), z})$ in the plane $\operatorname{span}(v(\theta), z)$, are all in $A$. If $\theta \in \Theta$, the angle is an not a rational

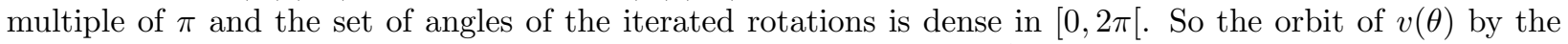
rotation and its iterates is dense in the one-dimensional sphere $S^{n-1} \cap \operatorname{span}(v(\theta), z)$. Since $A$ is closed, it follows that $S^{n-1} \cap \operatorname{span}(v(\theta), z) \subset A$. As it is possible to find a sequence in $\Theta$ converging to 0 , the closedness of $A$ yields

$$
S^{n-1} \cap \operatorname{span}(v, z) \subset A .
$$

This was proved for an arbitrary $v \in E \cap S^{n-1}$, hence we deduce that $S^{n-1} \cap \operatorname{span}(E, z) \subset A$, and we have found an isometric copy of $S^{k+1}$ in $A$. This completes the induction proof.

The following fact will be needed in order to estimate the volume product of a product of simplices.

Lemma 19. Consider integers $n_{1}, \ldots, n_{k} \geq 1$ and set $n=n_{1}+\cdots+n_{k}$. Then

$$
\prod_{\ell=1}^{k} \mathcal{P}\left(\Delta_{n_{\ell}}\right) n_{\ell} ! \geq \mathcal{P}\left(\Delta_{n}\right) n !
$$

with equality if and only if $k=1$.

Proof. Let $k \geq 2$. Since $\mathcal{P}\left(\Delta_{m}\right)=(m+1)^{m+1} /(m !)^{2}$, our task is to show that the function $\varphi(m)=$ $(m+1)^{m+1} / m$ ! defined for $m \in \mathbb{N}$ verifies $\varphi\left(n_{1}+\cdots+n_{k}\right)<\prod_{\ell=1}^{k} \varphi\left(n_{\ell}\right)$. By induction on $k$, it is enough to show that for all integers $p, q \geq 1$ we have $\varphi(p+q)<\varphi(p) \varphi(q)$. Since $\varphi(0)=1$, this may be written as

$$
\frac{\log \varphi(p+q)-\log \varphi(q)}{p}<\frac{\log \varphi(p)-\log \varphi(0)}{p} .
$$

From a classical convexity's argument on $\mathbb{R}$, it is enough to prove that the extension of $\varphi$ on $\mathbb{R}_{+}$defined by $\varphi(p)=(p+1)^{p+1} / \Gamma(p+1)$, for $p \in \mathbb{R}_{+}$, is strictly log-concave on $\mathbb{R}_{+}$. To see this, note that, with the change of variable $t=p u$, we get

$$
\frac{\Gamma(p)}{p^{p}}=\frac{1}{p^{p}} \int_{0}^{+\infty} t^{p} e^{-t} \frac{d t}{t}=\int_{0}^{+\infty}\left(u e^{-u}\right)^{p} \frac{d u}{u}=\|f\|_{L_{p}(\nu)}^{p},
$$

where $f(u)=u e^{-u}$ and $d \nu=\mathbf{1}_{(0,+\infty)} d u / u$. From Hölder's inequality we deduce that $p \mapsto \Gamma(p) / p^{p}$ is strictly $\log$-convex and therefore that $\varphi$ is strictly log-concave.

Proof of Theorem 16. The barycenter $b_{K}$ of $K$ is stable by all the symmetries of $K$. Hence $\cap_{i} H_{i}=\left\{b_{K}\right\}$. By translation invariance of the quantities of interest, we may assume that $b_{K}=0$. In this case the symmetries $S_{i}$ are linear maps. Let us show that without loss of generality, we may assume that they are also orthogonal. This is due to the fact that compact subgroups of the linear group are conjugated to subgroups of the orthogonal group. More precisely, let

$$
G=\left\{g \in \mathcal{L}\left(\mathbb{R}^{n}\right) ; g(K)=K\right\} .
$$

It is clearly a group $\left(0=b_{K} \in \operatorname{Int}(\mathrm{K})\right.$ since $K$ has non-empty interior, so any $g \in G$ is surjective). It is also closed as $K$ is. Eventually it is bounded since the inclusion $g(K-K) \subset K-K$ means that it is included in the unit ball of the set of linear endomorphism of the normed space $\left(\mathbb{R}^{n},\|\cdot\|_{K-K}\right)$. It follows that $G$ is a compact group. One may thus consider the scalar product on $\mathbb{R}^{n}$

$$
((x, y)):=\int_{G}\langle g x, g y\rangle d \mu(g),
$$


where $\mu$ is the bi-invariant Haar probability measure on $G$. It may be represented as $((x, y))=\langle A x, y\rangle$ for some symmetric positive matrix $A \in \mathcal{S}^{+}\left(\mathbb{R}^{n}\right)$. For all $g \in G,((g x, g y))=((x, y))$, meaning that $G$ is a subgroup of the orthogonal group for this new Euclidean structure. The latter isometry condition translates as $A={ }^{t} g A g$ for all $g \in G$. This implies that $A^{1 / 2} g A^{-1 / 2} \in \mathcal{O}\left(\mathbb{R}^{n}\right)$. Define $\tilde{K}=A^{1 / 2}(K)$. Then for all $g \in G, A^{1 / 2} g A^{-1 / 2} \tilde{K}=\tilde{K}$. Since the volume product is affine invariant, $\mathcal{P}(K)=\mathcal{P}(\tilde{K})$ and $\tilde{K}$ is invariant by the orthogonal symmetries $A^{1 / 2} S_{i} A^{-1 / 2}$ with respect to the hyperplanes $A^{1 / 2} H_{i}$.

From now on, we assume as we may, that the hyperplane symmetries $S_{1}, \ldots, S_{m}$ belong to $\mathcal{O}\left(\mathbb{R}^{n}\right)$. Let $\mathcal{R}(K)$ be the subgroup of $\mathcal{O}\left(\mathbb{R}^{n}\right)$ generated by all the hyperplane symmetries which leave $K$ globally invariant. By hypothesis $\operatorname{Fix}(\mathcal{R}(K)) \subset \bigcap_{i} H_{i}=\{0\}$. Hence by Proposition 17, there exists an orthogonal decomposition $\mathbb{R}^{n}=\bigoplus_{\ell=1}^{k} E_{\ell}$ and polytopes $P_{\ell} \in E_{\ell}$ such that $P_{\ell}$ is a regular polygon $\mathcal{C}_{m_{\ell}}$ if $n_{\ell}:=\operatorname{dim}\left(E_{\ell}\right)=2$ and $P_{\ell}$ is a regular simplex otherwise, such that

$$
\mathcal{O}\left(P_{1}\right) \times \cdots \times \mathcal{O}\left(P_{k}\right) \subset \mathcal{R}(K)
$$

By Theorem 13 and Lemma 14,

$$
\mathcal{P}(K) \geq \mathcal{P}\left(P_{1} \times \cdots \times P_{k}\right)=\frac{\prod_{\ell=1}^{k} n_{\ell} ! \mathcal{P}\left(P_{\ell}\right)}{n !} .
$$

If $n_{\ell}=\operatorname{dim}\left(E_{\ell}\right)=2$, then by Remark $2, \mathcal{P}\left(P_{\ell}\right)=\mathcal{P}\left(\mathcal{C}_{m_{\ell}}\right) \geq \mathcal{P}\left(\mathcal{C}_{3}\right)=\mathcal{P}\left(\Delta_{n_{\ell}}\right)$. Combining this observation with Lemma 19 yields the claim:

$$
\mathcal{P}(K) \geq \frac{\prod_{\ell=1}^{k} n_{\ell} ! \mathcal{P}\left(\Delta_{n_{\ell}}\right)}{n !} \geq \mathcal{P}\left(\Delta_{n}\right)
$$

Eventually we analyse the equality case. Assume that $P(K)=P\left(\Delta_{n}\right)$; necessarily the previous inequalities are equalities. Whenever $n_{\ell}=2$, one gets $\mathcal{P}\left(\mathcal{C}_{m_{\ell}}\right)=\mathcal{P}\left(\mathcal{C}_{3}\right)$ which forces $P_{\ell}$ to be a simplex as well. Next the equality condition in Lemma 19 yields $k=1$. This means that $K$ is $\mathcal{O}\left(\Delta_{n}\right)$-invariant and the equality condition of Theorem 10 allows to conclude that $K$ is a simplex.

\section{$7 \quad$ Functional forms and bodies of revolution}

Let $k, n_{1}, \ldots, n_{k} \geq 1$ be integers, let $n=n_{1}+\cdots+n_{k}$, let $L$ be an unconditional convex body in $\mathbb{R}^{k}$ and $K_{1} \subset \mathbb{R}^{n_{1}}, \ldots, K_{k} \subset \mathbb{R}^{n_{k}}$ be centrally symmetric convex bodies. Then the $L$-sum of $K_{1}, \ldots, K_{k}$ is defined as the convex body in $\mathbb{R}^{n}$

$$
\bigoplus_{L} K_{i}:=\left\{x=\left(x_{1}, \ldots, x_{k}\right) \in \mathbb{R}^{n_{1}} \times \cdots \times \mathbb{R}^{n_{k}} ;\left(\left\|x_{1}\right\|_{K_{1}}, \ldots,\left\|x_{k}\right\|_{K_{k}}\right) \in L\right\} .
$$

In [23], Saint Raymond proved that

$$
\mathcal{P}\left(\bigoplus_{L} K_{i}\right)=\int_{L \cap \mathbb{R}_{+}^{n}} \prod_{i=1}^{k} x_{i}^{n_{i}-1} d x \int_{L^{\circ} \cap \mathbb{R}_{+}^{n}} \prod_{i=1}^{k} x_{i}^{n_{i}-1} d x \prod_{i=1}^{k} n_{i} \mathcal{P}\left(K_{i}\right) \geq \mathcal{P}\left(\bigoplus_{B_{\infty}^{k}} K_{i}\right)=\frac{\prod_{i=1}^{k} n_{i} ! \mathcal{P}\left(K_{i}\right)}{n !} .
$$

This result is more general than Mahler's inequality for unconditional bodies (which correspond to $K_{i}=$ $[-1,1])$. In fact, using the above integral expression of $\mathcal{P}\left(\bigoplus_{L} K_{i}\right)$, it can be deduced from the lower bound of the volume product of unconditional bodies, applied to $\bigoplus_{L} B_{\infty}^{n_{i}}$ :

$$
\mathcal{P}\left(\bigoplus_{L} K_{i}\right)=\mathcal{P}\left(\bigoplus_{L} B_{\infty}^{n_{i}}\right) \times \prod_{i=1}^{k} \frac{\mathcal{P}\left(K_{i}\right)}{\mathcal{P}\left(B_{\infty}^{n_{i}}\right)} \geq \frac{4^{n}}{n !} \times \prod_{i=1}^{k} \frac{\mathcal{P}\left(K_{i}\right)}{\mathcal{P}\left(B_{\infty}^{n_{i}}\right)}=\frac{\prod_{i=1}^{k} n_{i} ! \mathcal{P}\left(K_{i}\right)}{n !} .
$$

The above result relates to ours, since $\bigoplus_{L} K_{i}$ is $\mathcal{O}\left(K_{i}\right) \times \cdots \times \mathcal{O}\left(K_{k}\right)$-invariant, but of a very specific form. Indeed, the $L$-sum of the convex bodies $K_{1}, \ldots, K_{k}$ may be seen as a body of revolution in the 
following generalized sense: for $x=\left(x_{1}, \ldots, x_{k-1}\right) \in \mathbb{R}^{k-1}$ such that $\left(x_{1}, \ldots, x_{k-1}, 0\right) \in L$, we define $f(x)=\left|\left\{t \in \mathbb{R}_{+} ;\left(x_{1}, \ldots, x_{k-1}, t\right) \in L\right\}\right|$. The function $f$ is unconditional, concave on its support and

$$
\bigoplus_{L} K_{i}=\left\{x=\left(x_{1}, \ldots, x_{k}\right) \in \mathbb{R}^{n_{1}} \times \cdots \times \mathbb{R}^{n_{k}} ;\left\|x_{k}\right\|_{K_{k}} \leq f\left(\left\|x_{1}\right\|_{K_{1}}, \ldots,\left\|x_{k-1}\right\|_{K_{k-1}}\right)\right\} .
$$

Hence Saint Raymond's generalization also implies a Mahler-type inequality for bodies of revolution built from unconditional functions. Let us see how to generalize this to functions with more general symmetries. Equivalently, as in $[16,5,6,7]$, this will also give some functional forms of Mahler's conjecture for concave functions having enough symmetries. First let us present the duality that we shall use for concave functions.

Let $n, m \geq 1$ be integers. For any convex body $L \subset \mathbb{R}^{m}$ with the origin in its interior its gauge function is defined by $\|x\|_{L}=\inf \{t>0 ; x \in t L\}$. To any non-negative concave function $f$ defined on a closed convex set $S \subset \mathbb{R}^{n}$, we associate $L(f)=\left\{(x, y) \in S \times \mathbb{R}^{m} ;\|y\|_{L} \leq f(x)\right\}$, the convex body of revolution constructed from $f$ with basis $L$. By Fubini's theorem, $|L(f)|=|L| \int_{S} f^{m}$ and the polar body of $L(f)$ is

$$
\begin{aligned}
L(f)^{\circ} & =\left\{\left(x^{\prime}, y^{\prime}\right) ;\left\langle x, x^{\prime}\right\rangle+\left\langle y, y^{\prime}\right\rangle \leq 1, \forall(x, y) \in L(f)\right\} \\
& =\left\{\left(x^{\prime}, y^{\prime}\right) ; f(x)\left\|y^{\prime}\right\|_{L^{\circ}} \leq 1-\left\langle x, x^{\prime}\right\rangle, \forall x \in S\right\} \\
& =\left\{\left(x^{\prime}, y^{\prime}\right) \in S^{\circ} \times \mathbb{R}^{m} ;\left\|y^{\prime}\right\|_{L^{\circ}} \leq f^{*}\left(x^{\prime}\right)\right\} \\
& =L^{\circ}\left(f^{*}\right),
\end{aligned}
$$

where $f^{*}: S^{\circ} \rightarrow \mathbb{R}_{+}$is the concave function defined by $f^{*}\left(x^{\prime}\right)=\inf _{f(x)>0} \frac{1-\left\langle x, x^{\prime}\right\rangle}{f(x)}$. Hence one has the equality:

$$
|L(f)|\left|L(f)^{\circ}\right|=|L|\left|L^{\circ}\right| \int f^{m} \int\left(f^{*}\right)^{m} .
$$

Using the case $L=B_{\infty}^{m}$, one deduces the following proposition.

Proposition 20. Let $n \geq 1$ and $m \geq 0$ be integers, let $L \subset \mathbb{R}^{m}$ be a convex body with Santaló point at the origin, let $S$ be a closed convex set in $\mathbb{R}^{n}$, let $f: S \rightarrow \mathbb{R}_{+}$be a concave function, let $\mathcal{O}(f)=\{T \in$ $\left.\mathcal{O}\left(\mathbb{R}^{n}\right) ; f(T x)=f(x), \forall x \in S\right\}$ and assume that $\operatorname{Fix}(\mathcal{O}(f))=\{0\}$. Let $L(f)=\left\{(x, y) \in S \times \mathbb{R}^{m} ;\|y\|_{L} \leq\right.$ $f(x)\}$ be the convex body of revolution built from $f$ with basis $L$. Then

$$
\mathcal{P}(L(f))=\frac{\mathcal{P}(L)}{\mathcal{P}\left(B_{\infty}^{m}\right)} \mathcal{P}\left(B_{\infty}^{m}(f)\right)
$$

Moreover $B_{\infty}^{m}(f)$ is $\mathcal{O}(f) \times \mathcal{O}\left(B_{\infty}^{m}\right)$-invariant.

Hence if $f$ is $G$-invariant for some subgroup $G$ of $\mathcal{O}\left(\mathbb{R}^{n}\right)$ with $\operatorname{Fix}(G)=\{0\}$ and if one has a lower bound on the volume product of the $G \times \mathcal{O}\left(B_{\infty}^{m}\right)$-invariant convex bodies then one gets a lower bound on the volume product of $L(f)$. From Theorem 13, we may already apply this argument for $G=\mathcal{O}\left(P_{1}\right) \times \cdots \times \mathcal{O}\left(P_{k}\right)$, for some regular polytopes $P_{1} \subset \mathbb{R}^{n_{1}}, \ldots, P_{k} \subset \mathbb{R}^{n_{k}}$, with $n_{1}+\cdots+n_{k}=n$. But we may also use the same argument to prove a bit more.

Actually let $K$ be a convex body in $\mathbb{R}^{n+m}=\mathbb{R}^{n} \times \mathbb{R}^{m}$ which is $G \times \mathcal{O}\left(\mathbb{R}^{m}\right)$-invariant, where $G$ is any subgroup of $\mathcal{O}\left(\mathbb{R}^{n}\right)$, with $\operatorname{Fix}(G)=\{0\}$. Let $S$ be the projection of $K$ on $\mathbb{R}^{n}$. For every $x \in S$, the section $K_{x}:=\left\{y \in \mathbb{R}^{m} ;(x, y) \in K\right\}$ is $\mathcal{O}\left(\mathbb{R}^{m}\right)$-invariant hence it is a Euclidean ball centered at the origin of radius $f(x)$, where $f: S \rightarrow \mathbb{R}^{+}$is defined by $f(x)=\left(\left|K_{x}\right| /\left|B_{2}^{m}\right|\right)^{1 / m}$. By Brunn's theorem, $f$ is concave. Moreover

$$
K=\left\{(x, y) \in S \times \mathbb{R}^{m} ;|y| \leq f(x)\right\}=B_{2}^{m}(f)
$$

and since $K$ is $G \times \mathcal{O}\left(\mathbb{R}^{m}\right)$-invariant, it follows that $f$ is $G$-invariant. Applying Proposition 20 we get that

$$
\mathcal{P}(K)=\mathcal{P}\left(B_{2}^{m}(f)\right)=\frac{\mathcal{P}\left(B_{2}^{m}\right)}{\mathcal{P}\left(B_{\infty}^{m}\right)} \mathcal{P}\left(B_{\infty}^{m}(f)\right),
$$


where $B_{\infty}^{m}(f)$ is $G \times \mathcal{O}\left(B_{\infty}^{m}\right)$-invariant. Therefore we reduced the problem of bounding from below the volume product of a $G \times \mathcal{O}\left(\mathbb{R}^{m}\right)$-invariant convex body to the same question for a $G \times \mathcal{O}\left(B_{\infty}^{m}\right)$-invariant convex body. By induction, we deduce that Theorem 13 extends to the following.

Theorem 21. Let $k \geq 1$ be an integer. For $1 \leq \ell \leq k$, let $P_{\ell}$ in $\mathbb{R}^{n_{\ell}}$ be a regular polytope or a Euclidean ball with barycenter at the origin. Let $n=\sum_{\ell} n_{\ell}$ and $K \subset \mathbb{R}^{n}=\mathbb{R}^{n_{1}} \times \cdots \times \mathbb{R}^{n_{k}}$ be a convex body invariant by $\mathcal{O}\left(P_{1}\right) \times \cdots \times \mathcal{O}\left(P_{k}\right)$. Then

$$
\mathcal{P}(K) \geq \mathcal{P}\left(P_{1} \times \cdots \times P_{k}\right)=\frac{1}{n !} \prod_{\ell=1}^{k} n_{\ell} ! \mathcal{P}\left(P_{\ell}\right) .
$$

Combining Theorem 21 with Proposition 20 yields the following functional form.

Corollary 22. Let $n \geq 1$ and $m \geq 0$ be integers, let $S$ be a closed convex set in $\mathbb{R}^{n}, f: S \rightarrow \mathbb{R}_{+}$ be a concave function and let $L \subset \mathbb{R}^{m}$ be a convex body with Santalo point at the origin. Let $L(f)=$ $\left\{(x, y) \in S \times \mathbb{R}^{m} ;\|y\|_{L} \leq f(x)\right\}$ be the convex body of revolution built from $f$ with basis L. If $\mathcal{O}(f)$ contains $\mathcal{O}\left(P_{1}\right) \times \cdots \times \mathcal{O}\left(P_{k}\right)$ for some regular polytopes or Euclidean balls $P_{1} \subset \mathbb{R}^{n_{1}}, \ldots, P_{k} \subset \mathbb{R}^{n_{k}}$, with $n_{1}+\cdots+n_{k}=$ $n$ then

$$
\mathcal{P}(L(f)) \geq \mathcal{P}\left(L \times P_{1} \times \cdots \times P_{k}\right) \quad \text { and } \quad \int f^{m} \int\left(f^{*}\right)^{m} \geq \frac{\mathcal{P}\left(P_{1} \times \cdots \times P_{k}\right)}{\left(\begin{array}{c}
n+m \\
n
\end{array}\right)} .
$$

Notice that in Corollary 22, there is equality for example if $f$ is the indicatrix of a cube or more generally the indicatrix of a Hanner polytope (see the end of section 5 above).

\section{Final remarks}

It is natural to ask for a version of Theorem 16 which would confirm the Mahler conjecture for origin symmetric convex sets having many hyperplane symmetries. We state it below and explain, in the course of the proof, that it is just a reformulation of Saint Raymond's result for unconditional sets.

Theorem 23. Let $K \subset \mathbb{R}^{n}$ be a convex body. Assume that there exist $m \geq 1$, and vector hyperplanes $H_{1}, \ldots, H_{m}$ such that for all $i \leq m, S_{H_{i}} K=K$ and $-\operatorname{Id}_{\mathbb{R}^{\mathrm{n}}}$ belongs to the group generated by these reflections. Then

$$
\mathcal{P}(K) \geq \mathcal{P}\left(B_{\infty}^{n}\right) .
$$

Proof. We simply reproduce the scheme of the proof of Proposition 17 and use the additional assumption that $-\operatorname{Id}_{\mathbb{R}^{\mathrm{n}}} \in \mathcal{R}(K)$ (the group generated by the reflections which leave $K$ globally invariant). We write $\mathcal{R}(K)$ as a direct product of irreducible reflection groups $\mathcal{R}_{i}$ acting on $\mathbb{R}^{n_{i}}$. Since $-\operatorname{Id}_{\mathbb{R}^{\mathrm{n}}} \in \mathcal{R}(K)$, it follows that for all $i,-\operatorname{Id}_{\mathbb{R}^{\mathrm{n}_{\mathrm{i}}}} \in \mathcal{R}_{i}$. Next we go through the list of possibilities for $\mathcal{R}_{i}$ and check that since $-\mathrm{Id}$ is in it, the group contains a product of $n_{i}$ copies of $\mathcal{O}([-1,1])$. The conclusion will be that $\mathcal{R}(K)$ contains a product of $n$-copies of $\mathcal{O}([-1,1])$. This means that $K$ is unconditional. So we will conclude by Saint Raymond's theorem, which is a particular case of Theorem 13.

We list below only the cases of irreducible reflection groups for which we have not already proved that they contain reflections with respect to the coordinates hyperplanes in some orthogonal basis. We check that either they contain such unconditional symmetries or they do not contain -Id:

- We have seen in Remark 2 that when a regular polygon $\mathcal{C}_{m}$ is origin symmetric if and only if $m$ is even, in which case it is unconditional. So we are done with this case.

- The regular simplex $\Delta_{n}$ is origin symmetric if and only if $n=1$. So the only possibility for such a factor is to be $\mathcal{O}([-1,1])$.

- The group $E_{6}$ is the group of isometries of a so-called Gosset polytope, with 27 vertices. Hence $-\operatorname{Id}_{\mathbb{R}^{6}} \notin E_{6}$. 
- From the definition, the demihypercube $D H^{n}$ is origin symmetric if and only if $n$ is even. In this case we have checked that it is unconditional.

As a final comment to this proof, let us point out that it is presumably possible to show that a reflection group containing minus the identity contains unconditional symmetries without using the classification, but simpler tools as the substitution rule for shortest representations and their product.

The method used in this paper is in a sense rather undirect. The natural approach would be, starting with a convex set $K \subset \mathbb{R}^{n}$ such that its reflection group fixes only the origin, to divide $\mathbb{R}^{n}$ into images of the fundamental domain $C$ of the group $\mathcal{R}(K)$. Note that $C$ is a polyhedral cone, bounded by hyperplanes of symmetry for $K$. The main difficulty of this approach is that it would lead to follow Meyer's volumic method in rather irregular cones. The argument seems to break in several places. For instance, the lower bound of $|K \cap C|\left|K^{\circ} \cap C\right|$ involves mixed terms of the form $\left|K \cap C_{i}\right|\left|K^{\circ} \cap C_{j}\right|$ where $C_{i}$ are the facets of $C$. These terms are not suited to induction when $i \neq j$. The additional structure of products of regular polytopes allowed to get rid of these terms.

Nevertheless it would be very interesting to find the exact minimum in Theorem 16 for a given set of hyperplane symmetries with only one common fixed point. These symmetries generate a subgroup of the linear group which is conjugated to a compact reflection group so that the question amounts to extend Theorem 21 to more general groups, i.e. to find the exact minimum of the volume product of the convex bodies which are invariant by a compact reflection group $G$ with $\operatorname{Fix}(G)=\{0\}$. As seen above, such a reflection group may be decomposed as a direct product of irreducible compact reflection groups, which are by Coxeter's classification, of the form $G=\mathcal{O}\left(P_{1}\right) \times \cdots \times \mathcal{O}\left(P_{k}\right)$, with $P_{1} \subset \mathbb{R}^{n_{1}}, \ldots, P_{k} \subset \mathbb{R}^{n_{k}}$ being semi-regular polytopes, regular polytopes or Euclidean balls and $n_{1}+\cdots+n_{k}=n$. So far, we have studied the case where the $P_{i}$ 's are regular polytopes or Euclidean balls and we have proved that in this case the minimum is reached for $P_{1} \times \cdots \times P_{k}$. Notice that for the isometry group of semi-regular polytopes the question will be more delicate.

For example, if we consider the case where the $P_{i}$ 's may be regular polytopes or Euclidean balls or demihypercubes $D H_{i}^{n}$ in even dimension $n_{i}$ then let us show that the minimum will not be reached for $P_{1} \times \cdots \times P_{k}$ but for $P_{1}^{\prime} \times \cdots \times P_{k}^{\prime}$, where $P_{i}^{\prime}=P_{i}$, if $P_{i}$ is a regular polytope or a Euclidean ball but $P_{i}^{\prime}=B_{\infty}^{n_{i}}$, if $P_{i}$ is a demihypercube. For notational simplicity, we prove this for $k=1$ and $P_{1}=D H^{n}$ for $n=2 m>4$ (the general case is proved in the same manner). As already pointed out, $D H^{2 m}$ is unconditional. Hence an $\mathcal{O}\left(D H^{2 m}\right)$-invariant convex body $K \subset \mathbb{R}^{2 m}$ is also unconditional and thus verifies $\mathcal{P}(K) \geq \mathcal{P}\left(B_{\infty}^{2 m}\right)$. The latter is an equality when $K=B_{\infty}^{2 m}$, which is $\mathcal{O}\left(D H^{2 m}\right)$-invariant. Moreover, the fact that the minimum is not reached for the demihypercube comes from the calculation

$$
\mathcal{P}\left(D H^{n}\right)=\frac{(n-1) 4^{n}}{(n-2) n !}\left(1-\frac{2^{n-1}}{n !}\right)>\frac{4^{n}}{n !}=\mathcal{P}\left(B_{\infty}^{n}\right),
$$

for $n \geq 5$ (there is equality for $n=4$, as $D H^{4}$ is isometric to $2 B_{1}^{4}$ ). Let us sketch this calculation. Recall that $D H^{n}=\operatorname{conv}\left\{x \in\{-1,1\}^{n} ; \prod_{i=1}^{n} x_{i}=1\right\}$. The demihypercube is obtained from the cube by cutting off $2^{n-1}$ simplices whose vertices are a vertex $x \in\{-1 ; 1\}^{n}$ of the cube such that $\prod x_{i}=-1$ and its $n$ neighbours. They have a right angle at $x$ and the length of the edges through this vertex are 2 . Thus

$$
\left|D H_{n}\right|=2^{n}-2^{n-1} \times \frac{2^{n}}{n !}=2^{n}\left(1-\frac{2^{n-1}}{n !}\right) .
$$

The equation of the preceding cutting hyperplane is $\{z ;\langle z, x\rangle=n-2\}$ since the scalar product of $x$ with any of its neighbour is $n-2$. Hence $D H_{n}=B_{\infty}^{n} \bigcap\left\{z ;\langle z, x\rangle \leq n-2, \forall x \in\{-1 ; 1\}^{n}, \prod x_{i}=-1\right\}$. Thus

$$
D H_{n}^{\circ}=\operatorname{conv}\left(\left\{e_{1}, \cdots, e_{n},-e_{1}, \cdots,-e_{n}\right\} \bigcup\left\{\frac{x}{n-2} ; x \in\{-1 ; 1\}^{n}, \prod_{i} x_{i}=-1\right\}\right) .
$$


It follows that $D H_{n}^{\circ}$ is obtained from $B_{1}^{n}$ by gluing $2^{n-1}$ simplices on half of its facets. Each of these simplices have the corresponding facet as a facet and $\frac{x}{n-2}$ as opposite vertex. The distance between this vertex and

the opposite facet is $d=\frac{\|x\|_{2}}{n-2}-\frac{1}{\sqrt{n}}=\frac{2}{\sqrt{n}(n-2)}$. Hence the volume of this simplex is $\frac{2}{(n-2) n !}$. Therefore we get

$$
\left|D H_{n}^{\circ}\right|=\frac{2^{n}}{n !}+2^{n-1} \frac{2}{(n-2) n !}=\frac{2^{n}}{n !} \times \frac{n-1}{n-2} .
$$

This completes the calculation of the volume product of the demihypercube.

\section{References}

[1] W. Blaschke: Vorlesungen über Differentialgeometrie II, Springer Verlag (1923).

[2] J. Bourgain, V. D. Milman: New volume ratio properties for convex symmetric bodies in $R^{n}$. Invent. Math. 88, no. 2, 319-340 (1987).

[3] H. S. M. Coxeter: Discrete groups generated by reflections. Ann. of Math. (2) 35, no. 3, 588-621 (1934).

[4] H. S. M. Coxeter: Regular polytopes. Third edition. Dover Publications, Inc., New York, 1973.

[5] M. Fradelizi, M. Meyer: Increasing functions and inverse Santaló inequality for unconditional functions, Positivity 12, 407-420 (2008).

[6] M. Fradelizi, M. Meyer: Some functional inverse Santaló inequalities, Adv. Math. 218, 1430-1452 (2008).

[7] M. Fradelizi, M. Meyer: Functional inequalities related to Mahler conjecture, Monatsh. Math. 159, No. $1-2,13-25$ (2010).

[8] Y. Gordon, M. Meyer, S. Reisner: Zonoids with minimal volume-product - a new proof. Proc. Amer. Math. Soc. 104, 273-276 (1988).

[9] J. E. Humphreys: Reflection groups and Coxeter groups. Cambridge Studies in Advanced Mathematics, 29. Cambridge University Press, Cambridge (1990).

[10] J. Kim, S. Reisner: Local minimality of the volume-product at the simplex. To apper in Mathematika. Preprint available at http://arxiv.org/abs/1001.0217.

[11] G. Kuperberg: From the Mahler conjecture to Gauss linking integrals. Geom. Funct. Anal. 18, no. 3, 870-892 (2008).

[12] K. Mahler: Ein Übertragungsprinzip für konvexe Körper. Časopis Pěst. Mat. Fys. 68, 93-102 (1939).

[13] K. Mahler: Ein Minimalproblem für konvexe Polygone. Mathematica (Zutphen) B 7, 118-127 (1939).

[14] M. Meyer: Une caractérisation volumique de certains espaces normés de dimension finie. Israel J. Math. 55 , no. 3, 317-326 (1986).

[15] M. Meyer, A. Pajor: On the Blaschke-Santaló inequality. Arch. Math. (Basel) 55, no. 1, 82-93 (1990).

[16] M. Meyer, S. Reisner: Inequalities involving integrals of polar-conjugate concave functions. Monatsh. Math. 125, no. 3, 219-227 (1998).

[17] M. Meyer, S. Reisner: Shadow systems and volumes of polar convex bodies. Mathematika 53, no. 1, 129-148 (2006).

[18] F. Nazarov: Hörmander's proof of the Bourgain-Milman theorem. Preprint. 
[19] F. Nazarov, F. Petrov, D. Ryabogin, A. Zvavitch: A remark on the Mahler conjecture: local minimality of the unit cube. Duke Math. J. 154 (2010), no 3, 419-430.

[20] G. Pisier: The volume of convex bodies and Banach space geometry. Cambridge Tracts in Mathematics, 94. Cambridge University Press, Cambridge, 1989.

[21] S. Reisner: Zonoids with minimal volume-product, Math. Z. 192, 339-346 (1986).

[22] S. Reisner: Minimal volume product in Banach spaces with a 1-unconditional basis, J. London Math. Soc. 36, 126-136 (1987).

[23] J. Saint Raymond: Sur le volume des corps convexes symétriques. Séminaire d'Initiation à l'Analyse, 1980/1981, Publ. Math. Univ. Pierre et Marie Curie, Paris (1981).

[24] L. A. Santaló: An affine invariant for convex bodies of $n$-dimensional space. Portugaliae Math. 8, 155$161(1949)$.

Franck Barthe : Equipe de Statistique et Probabilités, Institut de Mathématiques de Toulouse (IMT) CNRS UMR 5219, Université Paul-Sabatier, 31062 Toulouse Cedex 9, FRANCE.

Matthieu Fradelizi : Université Paris-Est Marne-la-Vallée, Laboratoire d'Analyse et de Mathématiques Appliquées UMR 8050, 77454 Marne-la-Vallée Cedex 2, FRANCE. 